\title{
Fermionic and bosonic fluctuation-dissipation theorem from a deformed AdS holographic model at finite temperature and chemical potential
}

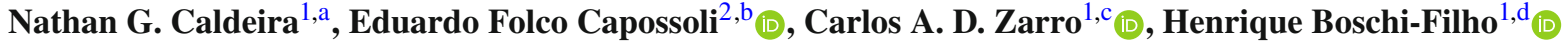 \\ ${ }^{1}$ Instituto de Física, Universidade Federal do Rio de Janeiro, 21.941-972 Rio de Janeiro, RJ, Brazil \\ ${ }^{2}$ Departamento de Física, Mestrado Profissional em Prááticas de Educação Básica (MPPEB), Colégio Pedro II, 20.921-903 Rio de Janeiro, RJ, \\ Brazil
}

Received: 18 September 2021 / Accepted: 21 December 2021 / Published online: 9 January 2022

(C) The Author(s) 2022

\begin{abstract}
In this work we study fluctuations and dissipation of a string in a deformed anti-de Sitter (AdS) space at finite temperature and density. The deformed AdS space is a charged black hole solution of the Einstein-MaxwellDilaton action. In this background we take into account the backreaction on the horizon function from an exponential deformation of the AdS space. From this model we compute the admittance and study the influence of the temperature and the chemical potential on it. We calculate the two-point correlations functions, and the mean square displacement for bosonic and fermionic cases, from which we obtain the short and large time approximations. For the long time, we obtain a sub-diffusive regime $\sim \log t$. Combining the results from the admittance and the correlations functions we check the fluctuation-dissipation theorem for bosonic and fermionic systems.
\end{abstract}

\section{Introduction}

Fluctuation and dissipation are ubiquitous phenomena in statistical physics. These two quantities are related by the famous fluctuation-dissipation theorem $[1,2]$. The natural set up to observe these properties is a system at finite temperature and density. In particular, ensembles at finite temperature combined with finite particle density captures a lot of attention from the high-energy community, specially for both high temperature and chemical potential. In this extreme environment, nuclear matter is totally deconfined, and is known as the quark-gluon plasma (QGP) [3-5]. By analyzing it, one

\footnotetext{
a e-mail: nathangomesc@ hotmail.com

be-mail: eduardo_capossoli@cp2.g12.br (corresponding author)

c e-mail: carlos.zarro@if.ufrj.br

de-mail: boschi@if.ufrj.br
}

can study from hadronic constituents to the interior of neutron stars. Many important experiments were idealized in order to reproduce on Earth these conditions as can be seen at relativistic heavy ion collider (RHIC) [6-9] and ALICE at CERN $[10,11]$. One can reasonably argue [12] that QGP can be understood within two different points of view: the thermodynamics and transport properties. Regarding the transport properties one can particularize their attention, for instance, on thermal fluctuations. An interesting review which relates QGP and fluctuations is presented in Ref. [13].

Concerned, in particular, with finite and high chemical potential, as we are dealing with an extreme environment, the calculations are very hard and usually it are not trustworthy. This means that lattice QCD starts to crash at baryonic chemical potential $\mu_{B} / T \gtrsim 1$ [14]. Such difficulties are usually related to an intrinsic characteristic of the canonical theoretical formulation known as the sign problem $[15,16]$. A recent and important progress in this direction has been done where an imaginary baryonic density was extrapolated to a real chemical potential around $\mu \sim 300 \mathrm{MeV}$ [17].

Among many proposals to tackle QCD, or even lattice QCD limitations, one can resort to the AdS/CFT correspondence, or in a broader sense string/gauge duality, originally proposed by Maldacena [18]. In one of its forms, it relates a weak coupling theory living in five-dimensional anti-de Sitter (AdS) space to a conformal $S U\left(N_{c} \rightarrow \infty\right) \mathcal{N}=4$ SYM QCD-like theory living in a four-dimensional Minkowski space. Mathematically speaking, such a duality can be stated since in supergravity (SUGRA) approximation of string theory in the AdS space, both theories can be related through $Z_{\mathrm{CFT}}\left[\varphi_{o}\right]=\left\langle\exp \left(\int_{\partial \Omega} d^{4} x \mathcal{O} \varphi_{o}\right)\right\rangle=\int_{\varphi_{o}} D \varphi \exp \left(-I_{S}(\varphi)\right)$ with $\varphi$ representing a non-normalizable SUGRA field, $I_{S}(\varphi)$ is the corresponding on shell SUGRA action, $\varphi_{o}$ is the value of $\varphi$ at the boundary $\partial \Omega$, and $\mathcal{O}$ is the associated operator of the conformal field theory (CFT). Making use of this cor- 
respondence many works were done in order to study hot dense QGP [19-24], considering finite chemical potentials (or some related topics as for instance, QCD phase transition, chiral symmetry breaking, critical exponents, etc. [25-39]) and quantum or thermal fluctuations such as the Brownian motion [40-46], fluctuation and dissipation [47-55], drag forces [56-60], or related topics [61-66].

Here in this work we will focus on the AdS/CFT correspondence to study holographically thermal fluctuations and dissipation modeled by a probe string attached to a probe brane in a deformed AdS-Reissner-Nördstrom (AdS-RN) spacetime with backreaction. Charged black holes, in the AdS/CFT scenario, were considered in the very first time in the Refs. [67,68]. The starting point of our model is an Einstein-Maxwell-Dilaton (EMD) string/gauge model at finite temperature and chemical potential. The holographic EMD and Einstein-Maxwell (EM) formulations, are wellknown ways to represent non-conformal plasmas and were considered in many works. An incomplete list, or even already cited throughout the text, can be seen in Refs. [6980].

Regarding the deformation in our AdS-RN spacetime we are considering, it can be understood as the introduction of a conformal exponential factor $\zeta(z)$, as a function of the holographic coordinate $z$, in the metric, written in the following form $[33,55,81]$ :

$$
\begin{aligned}
d s^{2} & =\frac{L^{2}}{\zeta(z)^{2}}\left(\frac{d z^{2}}{f(z)}-f(z) d t^{2}+d \vec{x}^{2}\right), \text { with } \\
\zeta(z) & =z e^{-\frac{1}{2}\left(k z^{2}\right)}
\end{aligned}
$$

where $k$ is a constant to be fixed latter and the AdS radius will be set as $L=1$. Notice that the introduction of this deformation was proposed in Refs. [82,83] as an alternative to the famous Softwall model (SWM) in Ref. [84], to break the conformal symmetry in order to generate a confining potential for a quark-antiquark pair. Besides, such a deformation in the AdS metric was successfully applied in different problems within string/gauge duality, as can be seen, for instance in Refs. [54,55,85-94]. If the limits $k \rightarrow 0$ and $f(z) \rightarrow 1$ are taken, one recovers the AdS spacetime in Poincaré coordinates.

It is worthwhile to mention that in this work the horizon function $f(z)$ will be properly achieved from the solutions of the EMD geometric background. This horizon function represents a deformed and backreacted AdS-ReissnerNördstrom (AdS-RN) spacetime. In this sense we will be taking into account backreaction contributions from the metric deformation and considering the string and the brane in the probe approximation.

Since we established the basic set up of our work, the reader will see it as in the following: In Sect.2, we state the fluctuation-dissipation theorem for bosons and fermions.
In Sect. 3, we present the mathematical description of the EMD geometry as well as the computation of our backreacted horizon function with finite chemical potential and the corresponding Hawking temperature. In Sect. 4, from the Nambu-Goto action we obtain the equations of motion for a string endpoint in this background, compute the admittance, the two point correlation functions, and the mean square displacement. For this last quantity we compute separately the bosonic $(\mu<0)$ and fermionic $(\mu>0)$ cases from which we obtain the short and long time behaviors. In Sect. 5, we check the fluctuation-dissipation theorem in this set up for bosons and fermions. Finally, in Sect. 6 we present our conclusions.

\section{Fluctuation-dissipation theorem}

In this section we are going to state the fluctuation-dissipation theorem, for the bosonic and fermionic cases which depend crucially on the sign of the chemical potential $\mu$.

First of all, let us define the symmetric Green's function in terms of the thermal correlation functions as:

$G_{\text {Sym }}(t) \equiv \frac{1}{2}(\langle x(t) x(0)\rangle+\langle x(0) x(t)\rangle)$.

For the bosonic case $(\mu<0)$, we have that the fluctuationdissipation theorem in the presence of a chemical potential can be written as [95]

$G_{\text {Sym }}(t)=\mathcal{F}^{-1}\left[\left(1+2 n_{B}\right) \Im \chi(\omega)\right]$,

and for the fermionic case $(\mu>0)$ one has [96]

$G_{\text {Sym }}(t)=\mathcal{F}^{-1}\left[\left(1+2 n_{F}\right) \Im \chi(\omega)\right]$,

where $\mathcal{F}^{-1}[\cdots]$ is the inverse Fourier transform, $n_{B}$ is the Bose-Einstein distribution, $n_{F}$ the Fermi-Dirac distribution, and $\Im \chi(\omega)$ is the imaginary part of the admittance.

In the following, we are going to define our holographic set up of a fluctuating string in a deformed AdS background with finite temperature and chemical potential from where we obtain the admittance and the correlation functions. As far as we are concerned, we could not find a discussion of the fluctuation-dissipation theorem in the holographic approach for bosons and fermions at finite density.

\section{The deformed and backreacted black hole}

Here, we will discuss our gravitational background starting from the EMD system. It was used in many references to deal with finite chemical potential. The action for this theory is written in the Einstein frame as: 


$$
\begin{aligned}
S= & \frac{1}{16 \pi G_{5}} \int d^{5} x \sqrt{-g}\left(R-\frac{4}{3} g^{m n} \partial_{m} \phi \partial_{n} \phi\right. \\
& \left.+V(\phi)-\frac{1}{4} F_{m n} F^{m n}\right)
\end{aligned}
$$

where $G_{5}$ is the 5-dimensional Newton's constant, $g$ is the metric determinant, $R$ is the Ricci scalar, $F_{m n}=\partial_{m} A_{n}-$ $\partial_{n} A_{m}$ is the Maxwell field, $\phi$ is the dilaton field, $V(\phi)$ its potential and $m, n=0,1,2,3,5$.

Thus, from this action one can obtain the following field equations:

$$
\begin{aligned}
& G_{m n}-\frac{4}{3}\left(\partial_{m} \phi \partial_{n} \phi-\frac{1}{2} g_{m n}(\partial \phi)^{2}\right) \\
& -\frac{1}{2} g_{m n} V(\phi)-\frac{1}{2}\left(F_{m a} F_{n}{ }^{a}-\frac{1}{4} g_{m n} F^{2}\right)=0, \\
& \square \phi+\frac{3}{8} \frac{\partial V(\phi)}{\partial \phi}=0, \\
& \nabla_{m} F^{m n}=0,
\end{aligned}
$$

where the Einstein tensor $G_{m n}$ can be written as

$$
G_{m n}=R_{m n}-\frac{1}{2} g_{m n} R
$$

In order to solve Eqs. (7), (8) and (9) we will used the metric given by Eq. (1), with the warp factor given by Eq. (2). Furthermore we will choose

$$
A_{m}(z)=\left(0, A_{t}(z), \overrightarrow{0}\right),
$$

where $A_{t}(z)$ is the time component of the $U(1)$ gauge field $A_{m}(z)$ which is dual to the presence of a global conserved current in the $4 D$ dual theory.

Replacing Eqs. (1) and (11) into Eqs. (7) and (9) one gets the set of coupled equations:

$$
\begin{aligned}
\frac{\zeta^{\prime \prime}(z)}{\zeta(z)}-\frac{4}{9} \phi^{\prime}(z)^{2} & =0, \\
\frac{\zeta^{\prime}(z)}{\zeta(z)}-\frac{A_{t}^{\prime \prime}(z)}{A_{t}^{\prime}(z)} & =0, \\
\frac{d}{d z}\left(\zeta(z)^{-3} f^{\prime}(z)\right)-\frac{A_{t}^{\prime}(z)^{2}}{\zeta(z)} & =0,
\end{aligned}
$$

where' represents derivative with respect to $z$. Solving Eq. (12), one obtains the dilaton field:

$$
\begin{aligned}
\phi(z)= & \pm \frac{3}{4}\left(\sqrt{k\left(k z^{2}-3\right)} z\right. \\
& \left.-3 \log \left(\frac{\sqrt{k\left(k z^{2}-3\right)}+k z}{C}\right)\right),
\end{aligned}
$$

where $C$ is a constant with units of energy. By using Eq. (8), one gets the dilaton potential:

$$
\begin{aligned}
V(\phi)= & 12 \zeta^{\prime}(z)^{2} f(z)-3 \zeta^{\prime}(z) f^{\prime}(z) \zeta(z) \\
& -\frac{4}{3} f(z) \zeta(z)^{2} \phi^{\prime}(z)^{2}+\frac{1}{2} \zeta(z)^{4} A_{t}^{\prime}(z)^{2} .
\end{aligned}
$$

Plugging the warp factor from Eq. (2) in Eq. (13) we get the following general solution for $A_{t}(z)$ :

$A_{t}(z)=-\frac{C_{1} e^{-\frac{1}{2}\left(k z^{2}\right)}}{k}+C_{2}$,

where $C_{1}$ and $C_{2}$ are constants to be fixed by imposing the regularity conditions at the horizon. These conditions are $A_{t}\left(z=z_{h}\right)=0$ and $A_{t}(z=0)=\mu$, where $\mu$ is the chemical potential of the dual gauge theory. Hence, after some algebra one gets the expression for $A_{t}(z)$, given by:

$A_{t}(z)=\mu\left(\frac{e^{\frac{1}{2} k\left(z_{h}^{2}-z^{2}\right)}-1}{e^{\frac{k}{2} z_{h}^{2}}-1}\right)$.

If one wants to recover the AdS-RN space, one can take the limit $k \rightarrow 0$, so that:

$A_{t}^{\mathrm{AdS}-\mathrm{RN}}(z)=\mu\left(1-\frac{z^{2}}{z_{h}^{2}}\right)$.

Now, using Eqs. (2) and (13), satisfying $f(0)=1$ (pure AdS ) and the horizon property $f\left(z_{h}\right)=0$, one can solve analytically Eq. (14), so that:

$$
\begin{aligned}
f(z)= & 1-\frac{\mathcal{G}(z)}{\mathcal{G}\left(z_{h}\right)} e^{\frac{3 k}{2}\left(z_{h}^{2}-z^{2}\right)}-\frac{\mu^{2} \mathcal{H}\left(z_{h}\right)}{72 k\left(e^{\frac{1}{2} k z_{h}^{2}}-1\right)^{2}} \\
& \times\left[\frac{\mathcal{G}(z)}{\mathcal{G}\left(z_{h}\right)} e^{\frac{k}{2}\left(z_{h}^{2}-3 z^{2}\right)}-\frac{\mathcal{H}(z)}{\mathcal{H}\left(z_{h}\right)} e^{k\left(z_{h}^{2}-2 z^{2}\right)}\right],
\end{aligned}
$$

where

$$
\begin{aligned}
\mathcal{G}(z) & \equiv 3 k z^{2}-2 e^{\frac{3}{2} k z^{2}}+2 \\
\mathcal{H}(z) & \equiv 18 k z^{2}+7 e^{2 k z^{2}}-24 e^{\frac{1}{2} k z^{2}} k z^{2}-16 e^{\frac{1}{2} k z^{2}}+9 .
\end{aligned}
$$

In Fig. 1 we present the behavior of the horizon function, coming from Eq. (20), in terms of the holographic coordinate $z$. Note that in all Figures of this work we use arbitrary units for the physical parameters $k, \mu$, etc.

Moreover, one can verify that horizon function $f(z)$ for the AdS-RN space is recovered for $k \rightarrow 0$ which is given by:

$$
f^{\mathrm{AdS}-\mathrm{RN}}(z)=1-\frac{z^{4}}{z_{h}^{4}}-\frac{\mu^{2} z_{h}^{2}}{3}\left(\frac{z^{4}}{z_{h}^{4}}-\frac{z^{6}}{z_{h}^{6}}\right),
$$

in agreement with the literature, see e.g., [97]. 

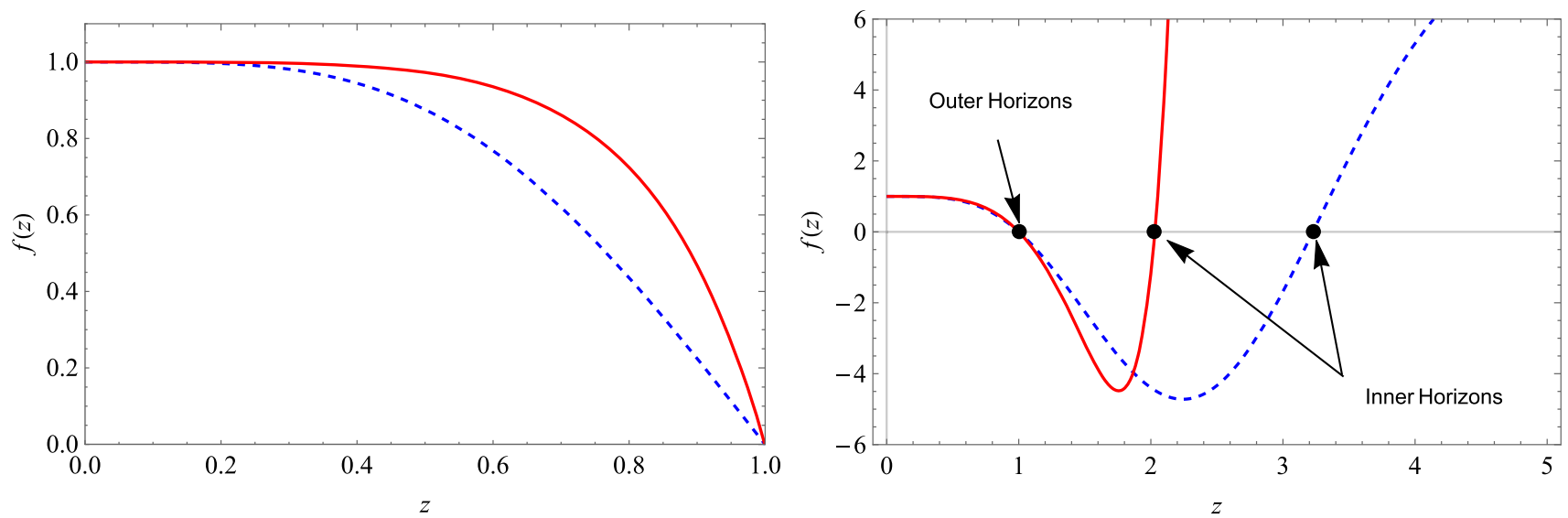

Fig. 1 Left panel: horizon function as a function o $z$ for $\mu=0$. The dashed blue curve represents $k=1$ and the red curve represents $k=-1$. Right panel: horizon function as a function of $z$ for $\mu=0.8$. The dashed blue curve represents $k=0.18$ and the red curve represents $k=-0.18$

\subsection{Hawking temperature}

In this subsection we will calculate the Hawking temperature associated with the black hole present in our deformed and backreacted AdS-RN spacetime which is the temperature of the bath at the boundary. Firstly, let us make a change of coordinates $r=1 / z$. In this coordinate system the horizon function Eq. (20) reads:

$$
\begin{aligned}
f(r)= & 1-\frac{\mathcal{G}(r)}{\mathcal{G}\left(r_{h}\right)} e^{\frac{3 k}{2}\left(r_{h}^{-2}-r^{-2}\right)}-\frac{\mu^{2} \mathcal{H}\left(r_{h}\right)}{72 k\left(e^{\frac{1}{2} k r_{h}^{-2}}-1\right)^{2}} \\
& \times\left[\frac{\mathcal{G}(r)}{\mathcal{G}\left(r_{h}\right)} e^{\frac{k}{2}\left(r_{h}^{-2}-3 r^{-2}\right)}-\frac{\mathcal{H}(r)}{\mathcal{H}\left(r_{h}\right)} e^{k\left(r_{h}^{-2}-2 r^{-2}\right)}\right]
\end{aligned}
$$

where

$$
\begin{aligned}
\mathcal{G}(r) & \equiv 3 k r^{-2}-2 e^{\frac{3}{2} k r^{-2}}+2 ; \\
\mathcal{H}(r) & \equiv 18 k r^{-2}+7 e^{2 k r^{-2}}-24 e^{\frac{1}{2} k r^{-2}} k r^{-2}-16 e^{\frac{1}{2} k r^{-2}}+9 .
\end{aligned}
$$

The Hawking temperature can be obtained from Eq. (23), so that:

$T=\left.\left|\frac{r^{2}}{4 \pi} \frac{d f(r)}{d r}\right|\right|_{r=r_{h}}=\frac{r_{h}}{\pi}\left|g(x)-\frac{\mu^{2}}{k} c(x)\right|$,

where $x \equiv k / r_{h}^{2}$,

$g(x) \equiv \frac{9 x^{2}}{4\left(2\left(e^{\frac{3}{2} x}-1\right)-3 x\right)}$ and

$$
c(x) \equiv \frac{e^{-\frac{3 x}{2}}\left(6 e^{x / 2} x^{3}+7 e^{x / 2} x^{2}+9 e^{\frac{5 x}{2}} x^{2}-16 e^{2 x} x^{2}\right)}{32\left(e^{x / 2}-1\right)^{2}\left(-3 x+2 e^{\frac{3 x}{2}}-2\right)} .
$$

Then, the allowed physical values for $\mu$ are

$$
\begin{aligned}
& -\sqrt{\frac{72 e^{x}\left(e^{x / 2}-1\right)^{2} x}{6 x-16 e^{\frac{3 x}{2}}+9 e^{2 x}+7}} \leq \frac{\mu}{r_{h}} \\
& \leq \sqrt{\frac{72 e^{x}\left(e^{x / 2}-1\right)^{2} x}{6 x-16 e^{\frac{3 x}{2}}+9 e^{2 x}+7}} .
\end{aligned}
$$

This expression represents constraints for the chemical potential $\mu$ and the constant $k$. It's worthwhile to mention that for $k \rightarrow 0$ one recovers

$$
-\sqrt{6} \leq \frac{\mu}{r_{h}} \leq \sqrt{6},
$$

as expected for AdS-RN black hole, as one can see in Ref. [97], where the author considered $\mu \geq 0$. In Fig. 2, we present the behavior of Hawking temperature as a function of $r_{h}$ for positive and negative values of $k$, with different values of the chemical potential.

\section{Admittance, correlation functions and mean square displacement}

In this section we will obtain the equations of motion from Nambu-Goto action and compute the main quantities related to the Brownian motion, such as, the admittance, the mean square displacement and the correlation functions. 


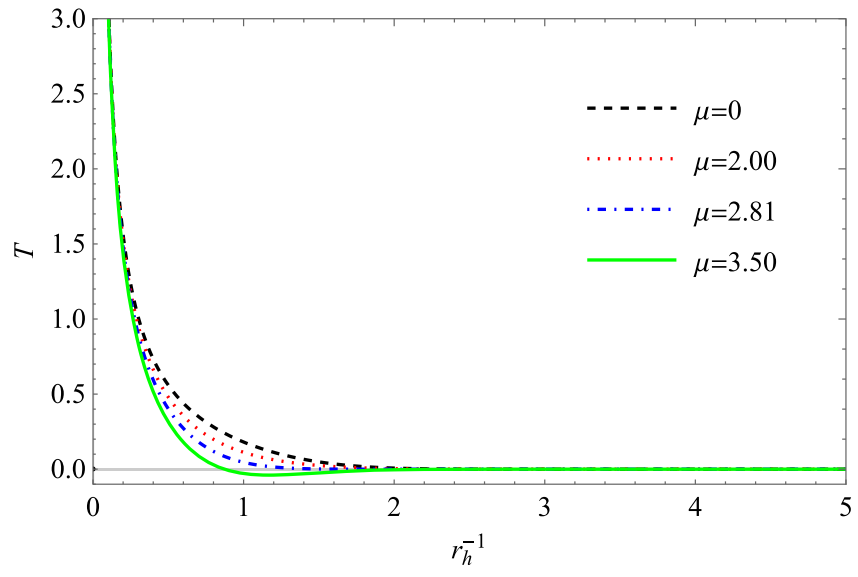

Fig. 2 Left panel: temperature versus $r_{h}^{-1}$ for a fixed $k=1$ and some values of $\mu$. The value $\mu=2.81$ corresponds to a critical chemical potential. For $\mu>\mu_{c}$ the temperature would become negative. Right

\subsection{Nambu-Goto action and equations of motion}

Here, we will consider our probe string attached to a probe brane in the EMD background. This system is dual to a particle in a thermal bath with a finite chemical potential. The metric of the deformed and backreacted AdS-RN spacetime Eq. (1), in the $r$ coordinate system, is written as:

$d s^{2}=e^{\frac{k}{r^{2}}}\left[-r^{2} f(r) d t^{2}+r^{2}\left(\eta_{i j} d x^{i} d x^{j}\right)+\frac{d r^{2}}{r^{2} f(r)}\right]$.

Besides, the dynamics of such a string will be given by the Nambu-Goto action:

$S_{N G}=-\frac{1}{2 \pi \alpha^{\prime}} \int d \tau d \sigma \sqrt{-\gamma}$,

where $\alpha^{\prime}$ is the Regge slope, $\gamma=\operatorname{det}\left(\gamma_{\alpha \beta}\right)$ and $\gamma_{\alpha \beta}=$ $g_{m n} \partial_{\alpha} X^{m} \partial_{\beta} X^{n}$ is the induced metric on the worldsheet with $m, n=0,1,2,3,5$.

We choose a static gauge, where $t=\tau, r=\sigma$ and $X=X(\tau, \sigma)$, as done in Refs. [48,53]. By using the metric, Eq. (30), and expanding the Nambu-Goto action, Eq. (31), in order to keep the quadratic terms $\dot{X}^{2}, X^{\prime 2}$, we get:

$S_{N G} \approx-\frac{1}{4 \pi \alpha^{\prime}} \int d \tau d \sigma\left[\dot{X}^{2} \frac{e^{\frac{k}{r^{2}}}}{f(r)}-X^{\prime 2} r^{4} f(r) e^{\frac{k}{r^{2}}}\right]$,

where $\dot{X}=\partial_{\tau=t} X$ and $X^{\prime}=\partial_{\sigma=r} X$.

The equation of motion is

$$
\frac{\partial}{\partial r}\left(r^{4} f(r) e^{\frac{k}{r^{2}}} X^{\prime}(t, r)\right)-\frac{e^{\frac{k}{r^{2}}}}{f(r)} \ddot{X}(t, r)=0,
$$

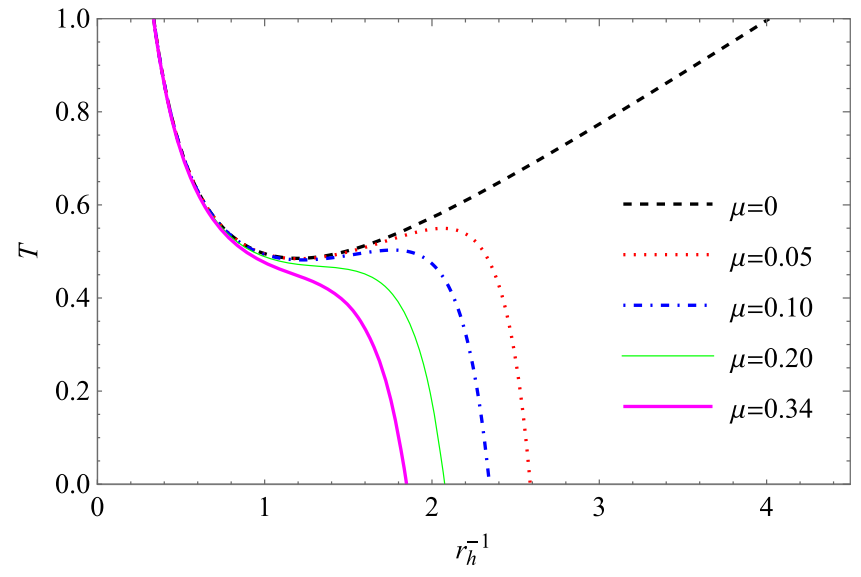

panel: temperature versus $r_{h}^{-1}$ for a fixed $k=-1$ and some values of $\mu$. The critical chemical potential is $\mu_{c}=0.34$. For $\mu>\mu_{c}$ the temperature only decreases

where $f(r)$ is given by Eq. (23) which can be expanded close to the horizon $\left(r \sim r_{h}\right)$ as $f(r) \approx f^{\prime}\left(r_{h}\right)\left(r-r_{h}\right)$. Using the ansatz $X(t, r)=e^{i \omega t} h_{\omega}(r)$, one gets:

$$
\begin{aligned}
& r^{4} f(r) h^{\prime \prime}(r)+\left[-2 k r f(r)+r^{4} f^{\prime}(r)+4 r^{3} f(r)\right] h^{\prime}(r) \\
& +\frac{\omega^{2}}{f(r)} h(r)=0
\end{aligned}
$$

Changing to a tortoise coordinate $r_{*}=\int d r\left(r^{2} f(r)\right)^{-1}$, we get

$\frac{1}{f(r)} \frac{d^{2} h_{\omega}\left(r_{*}\right)}{d r_{*}^{2}}+\left(-\frac{2 k}{r}+2 r\right) \frac{d h_{\omega}}{d r_{*}}+\frac{\omega^{2}}{f(r)} h_{\omega}(r)=0$.

Making a Bogoliubov transformation $h_{\omega}\left(r_{*}\right)=e^{B\left(r_{*}\right)} \psi\left(r_{*}\right)$, with $B(r)=-k /\left(2 r^{2}\right)-\log (r)$, we obtain a Schrödingerlike equation

$\frac{d^{2} \psi\left(r_{*}\right)}{d r_{*}^{2}}+\left(\omega^{2}-V(r)\right) \psi\left(r_{*}\right)=0$,

with potential

$V(r)=-f(r)\left[\left(-\frac{k^{2}}{r^{2}}+k-2 r^{2}\right) f(r)+r\left(k-r^{2}\right) f^{\prime}(r)\right]$

From the above equation, one has $V\left(r=r_{h}\right)=0$, since $f\left(r_{h}\right)=0$.

The Eq. (36) cannot be analytically solved, hence one seeks for approximate solutions. This is discussed in Appendix A. The relevant solution close to the boundary 
is:

$$
h_{\omega}^{C}(r) \approx A_{1}\left(\frac{e^{-\frac{k}{2 r_{h}^{2}}}}{r_{h}}+\frac{i \omega r_{h} e^{\frac{k}{2 r_{h}^{2}}}}{3 r^{3}}\right),
$$

where $A_{1}$ is a normalization constant.

\subsection{Admittance}

In this section, we will present one of the main results of this work, which is the admittance in the presence of a finite chemical potential in a backreacted geometry. From the solution given by Eq. (38), one can compute the the admittance $\chi(\omega)$, also known as the linear response, of the string endpoint on the brane. One should note that such a response is due to the action of an external force in an arbitrary brane direction, $x^{i}$, and can be represented by $F(\omega)=E e^{-i \omega t}$, where $E$ is the electric field on the brane.

By considering the electric field as $E=E\left(A_{t}, \overline{\hat{\imath}}\right.$ and taking into account the approximate Nambu-Goto action, one has:

$$
\begin{aligned}
S \approx & -\frac{1}{4 \pi \alpha^{\prime}} \int d \tau d \sigma\left[\dot{X}^{2} \frac{e^{\frac{k}{r^{2}}}}{f(r)}-X^{\prime 2} r^{4} f(r) e^{\frac{k}{r^{2}}}\right] \\
& +\left.\int d t\left(A_{t}+\vec{A} \cdot \overrightarrow{\dot{x}}\right)\right|_{r=r_{b}},
\end{aligned}
$$

where the second integral is a surface term. Such a term was chosen in an arbitrary direction, and it does not participate in the bulk dynamics. Choosing $A_{t}=0$ and integrating by parts the surface term, one gets:

$$
\begin{aligned}
S & \approx-\frac{1}{4 \pi \alpha^{\prime}} \int d t d r\left[\dot{X}^{2} \frac{e^{\frac{k}{r^{2}}}}{f(r)}-X^{\prime 2} r^{4} f(r) e^{\frac{k}{r^{2}}}\right] \\
& -\left.\int d t F(t)\left(\frac{\partial X(t, r)}{\partial r}\right)\right|_{r=r_{b}},
\end{aligned}
$$

with $\tau=t$ and $\sigma=r$. Computing $\delta S / \delta X^{\prime}=0$ and imposing Neumann boundary condition on the brane, one can write the force in a Fourier domain as:

$F(\omega)=\frac{A_{1}}{2 \pi \alpha^{\prime}}\left[-i \omega r_{h} e^{\frac{k}{2 r_{h}^{2}}} f\left(r_{b}\right) e^{\frac{k}{r_{b}^{2}}}\right]$.

More details on the calculation of $F(\omega)$ can be found with in Refs. [47,48,53-55].

Therefore the admittance is

$\chi(\omega) \equiv \frac{h_{\omega}^{C}(\omega)}{F(\omega)}=\frac{2 \pi i \alpha^{\prime}}{\omega r_{h}^{2} e^{\frac{k}{r_{h}^{2}}} f\left(r_{b}\right) e^{\frac{k}{r_{b}^{2}}}}\left(1+\frac{i \omega r_{h}^{2} e^{\frac{k}{r_{h}^{2}}}}{r_{b}^{3}}\right)$.
In the limit $r_{b} \gg r_{h}, r_{b} \gg k$ and $f\left(r_{b}\right) \rightarrow 1$ the admittance reads

$\chi(\omega)=-2 \pi \alpha^{\prime} \frac{e^{-\frac{k}{r_{h}^{2}}}}{i \omega r_{h}^{2}}=\frac{2 \pi i \alpha^{\prime} e^{-x}}{\omega r_{h}^{2}}$.

Note that the horizon radius $r_{h}$ is a function of the Hawking temperature, Eq. (25), so that

$r_{h}=\frac{\pi T}{\left|g(x)-\frac{\mu^{2}}{k} c(x)\right|}$

Then the admittance, Eq. (43), can be written as

$\chi(\omega)=\frac{2 i \alpha^{\prime} e^{-x}}{\omega \pi T^{2}}\left|g(x)-\frac{\mu^{2}}{k} c(x)\right|^{2}$,

where we recall that $x=k / r_{h}^{2}$, and then one can see that the admittance is a non-trivial function of the temperature $T$. This is one of the main results of this work. Note that this result is independent of the sign of the chemical potential although it will be relevant in the correlations functions to be calculated in the next section.

Using the fact that the admittance can be written as [53]

$\chi(\omega)=2 \pi \alpha^{\prime}\left(\frac{i}{\gamma \omega}-\frac{\Delta m}{\gamma^{2}}+\mathcal{O}(\omega)\right)$,

we find in our case

$$
\gamma=\frac{e^{-\frac{k}{r_{h}^{2}}}}{r_{h}^{2}}\left(1+\frac{k}{r_{b}^{2}}+O\left(\frac{k^{2}}{r_{b}^{4}}\right)\right)
$$

$\Delta m=\frac{r_{h}^{4} e^{\frac{2 k}{r_{h}^{2}}}}{r_{b}^{3}}\left(1+\frac{k}{r_{b}^{2}}+O\left(\frac{k^{2}}{r_{b}^{4}}\right)\right)$,

where $\gamma$ is the friction coefficient and $\Delta m$ corresponds to the change in the bare mass $m$ of the particle described by the Langevin equation [1,2] (see also [45]).

If one considers the admittance for case of the string in pure AdS-Schwarzschild, one finds [40]

$\chi_{A d S}(\omega)=\frac{2 i \alpha^{\prime}}{\omega \pi T^{2}}$.

In Refs. [54,55], the problem of a probe string immersed in a black hole metric deformed by an exponential conformal factor $\exp \left(k / r^{2}\right)$ was studied. Regarding backreaction effects, the Ref. [54] did not consider them and the admittance found was:

$\chi_{N B R}(\omega)=\frac{2 i \alpha^{\prime} e^{-x}}{\omega \pi T^{2}}, \quad$ (No Backreaction),

while in Ref. [55], taking into account backreaction, it was obtained that

$\chi_{B R}(\omega)=\frac{2 i \alpha^{\prime} e^{-x}}{\omega \pi T^{2}}(g(x))^{2}, \quad$ (Backreacted). 
Note that these results are particular cases of the admittance $\chi(\omega)$ computed in the present work, Eq. (45), where finite density and an exponential deformation with backreaction were included, so that

$$
\begin{aligned}
\chi(\omega) & =\chi_{A d S}(\omega)\left|g(x)-\frac{\mu^{2}}{k} c(x)\right|^{2} e^{-x} ; \\
& =\chi_{N B R}(\omega)\left|g(x)-\frac{\mu^{2}}{k} c(x)\right|^{2} ; \\
& =\chi_{B R}(\omega)\left|1-\frac{\mu^{2}}{k} \frac{c(x)}{g(x)}\right|^{2} .
\end{aligned}
$$

These equations show the influence of the chemical potential $\mu$ on the admittance $\chi(\omega)$. In particular, Eq. (54), displays this extension codified in the term $\mu^{2} c(x) / k g(x)$. The admittance given by Eqs. (45), (50), and (51) are depicted in Fig. 3. This picture shows that the presence of a finite chemical potential and its increase dislocates the curve of the imaginary part of the admittance towards low temperatures.

\subsection{Correlation functions and mean square displacement with chemical potential}

In this section, we will calculate the correlation functions and the mean square displacement of the string endpoint on the brane with non-zero chemical potential. Starting with the definition of the density operator in the grand canonical ensemble

$\rho_{0}=\frac{\left.e^{-\beta\left(\sum_{\omega>0} \omega a_{\omega}^{\dagger} a_{\omega}-\mu a_{\omega}^{\dagger} a_{\omega}\right.}\right)}{\operatorname{Tr}\left(e^{-\beta(H-\mu N)}\right)}$,

where the creation and annihilation operators satisfy

$\left\langle a_{\omega}^{\dagger} a_{\omega}\right\rangle=\frac{\delta_{\omega \omega^{\prime}}}{e^{\beta(\omega-\mu)} \mp 1} ; \quad\left\langle a_{\omega}^{\dagger} a_{\omega}^{\dagger}\right\rangle=0 ; \quad\left\langle a_{\omega} a_{\omega}\right\rangle=0$,

and the minus (plus) sign corresponds to the bosonic (fermionic) case, one can calculate the correlation function:

$$
\begin{aligned}
& \langle x(t) x(0)\rangle \equiv\left\langle X\left(t, r_{b}\right) X\left(0, r_{b}\right)\right\rangle \\
& =\sum_{\omega>0} \sum_{\omega^{\prime}>0}\left(\frac{h_{\omega}^{*}\left(r_{b}\right) h_{\omega^{\prime}}\left(r_{b}\right) e^{i \omega t}+h_{\omega}\left(r_{b}\right) h_{\omega^{\prime}}^{*}\left(r_{b}\right) e^{-i \omega t}}{e^{\beta(\omega-\mu)} \mp 1}\right. \\
& \left.+h_{\omega}\left(r_{b}\right) h_{\omega^{\prime}}^{*}\left(r_{b}\right) e^{-i \omega t}\right) \delta_{\omega \omega^{\prime}} \\
& =\frac{\pi \alpha^{\prime} r_{h}^{2} f^{\prime}\left(r_{h}\right)}{2 \log \left(\frac{1}{\epsilon}\right)} \sum_{\omega>0} \frac{1}{\omega} \frac{e^{-\frac{k}{r_{h}^{2}}}}{r_{h}^{2}}\left\{\left(1+\frac{\omega^{2} r_{h}^{4} e^{\frac{2 k}{r_{h}^{2}}}}{9 r_{b}^{6}}\right)\right. \\
& \left.+\Re\left[B^{*}\left(1+\frac{\omega^{2} r_{h}^{4} e^{\frac{2 k}{r_{h}^{2}}}}{9 r_{b}^{6}}-\frac{2 i \omega r_{h}^{2} e^{\frac{k}{r_{h}^{2}}}}{3 r_{b}^{3}}\right)\right]\right\}
\end{aligned}
$$

$$
\times\left(\frac{2 \cos (\omega t)}{e^{\beta(\omega-\mu)} \mp 1}+e^{-i \omega t}\right)
$$

where we used the solution in terms of the ingoing and outgoing modes $h_{\omega}(r)=A\left[h_{\omega}^{\text {out }}(r)+B h_{\omega}^{\text {in }}(r)\right]$ (see the Appendix B). Then, we get:

$$
\begin{aligned}
\langle x(t) x(0)\rangle= & \frac{\pi \alpha^{\prime} r_{h}^{2} f^{\prime}\left(r_{h}\right)}{\log \left(\frac{1}{\epsilon}\right)} \sum_{\omega>0} \frac{1}{\omega} \frac{e^{-\frac{k}{r_{h}^{2}}}}{r_{h}^{2}} \\
& \times\left(\frac{2 \cos (\omega t)}{e^{\beta(\omega-\mu)} \mp 1}+e^{-i \omega t}\right) .
\end{aligned}
$$

This sum can be approximated by an integral. For this purpose we use that $\Delta \omega=\pi r_{h}^{2} f^{\prime}\left(r_{h}\right) / \log \left(\epsilon^{-1}\right) \sim d \omega$, so that we write

$$
\begin{aligned}
\langle x(t) x(0)\rangle & =\frac{\alpha^{\prime} e^{-\frac{k}{r_{h}^{2}}}}{r_{h}^{2}} \int_{0}^{\infty} \frac{d \omega}{\omega}\left(\frac{2 \cos (\omega t)}{e^{\beta(\omega-\mu)} \mp 1}+e^{-i \omega t}\right) \\
& =\langle x(0) x(t)\rangle^{*} .
\end{aligned}
$$

In a similar way we get that

$$
\begin{aligned}
\langle x(t) x(t)\rangle & =\left\langle X\left(t, r_{b}\right) X\left(t, r_{b}\right)\right\rangle \\
& =\sum_{\omega>0}\left|h_{\omega}^{C}\left(r_{b}\right)\right|^{2}\left(\frac{2}{e^{\beta(\omega-\mu)} \mp 1}+1\right) \\
& =\frac{\alpha^{\prime} e^{-\frac{k}{r_{h}^{2}}}}{r_{h}^{2}} \int_{0}^{\infty} \frac{d \omega}{\omega}\left(\frac{2}{e^{\beta(\omega-\mu)} \mp 1}+1\right) \\
& =\langle x(0) x(0)\rangle
\end{aligned}
$$

Now, we can compute the regularized mean square displacement defined by:

$s_{\text {reg }}^{2}(t)=\left\langle:[x(t)-x(0)]^{2}:\right\rangle \equiv\left\langle:\left[X\left(t, r_{b}\right)-X\left(0, r_{b}\right)\right]^{2}:\right\rangle$,

where we used the notation $:[\cdots]:$ to represent the normal ordering prescription.

Plugging Eqs. (59), (60) and (61) into the definition of the mean square displacement, we find:

$$
\begin{gathered}
s_{\text {reg }}^{2}(t)=\frac{\alpha^{\prime} e^{-\frac{k}{r_{h}^{2}}}}{r_{h}^{2}} \int_{0}^{\infty} \frac{d \omega}{\omega}\left(\frac{4(1-\cos (\omega t))}{e^{\beta(\omega-\mu)} \mp 1}\right) \\
=\frac{8 \alpha^{\prime} e^{-\frac{k}{r_{h}^{2}}}}{r_{h}^{2}} \int_{0}^{\infty} \frac{d \omega}{\omega}\left(\frac{\sin ^{2}\left(\frac{\omega t}{2}\right)}{e^{\beta(\omega-\mu)} \mp 1}\right) .
\end{gathered}
$$

Up to now, we have been considering the bosonic and fermionic together. From now on, we will split this analy- 

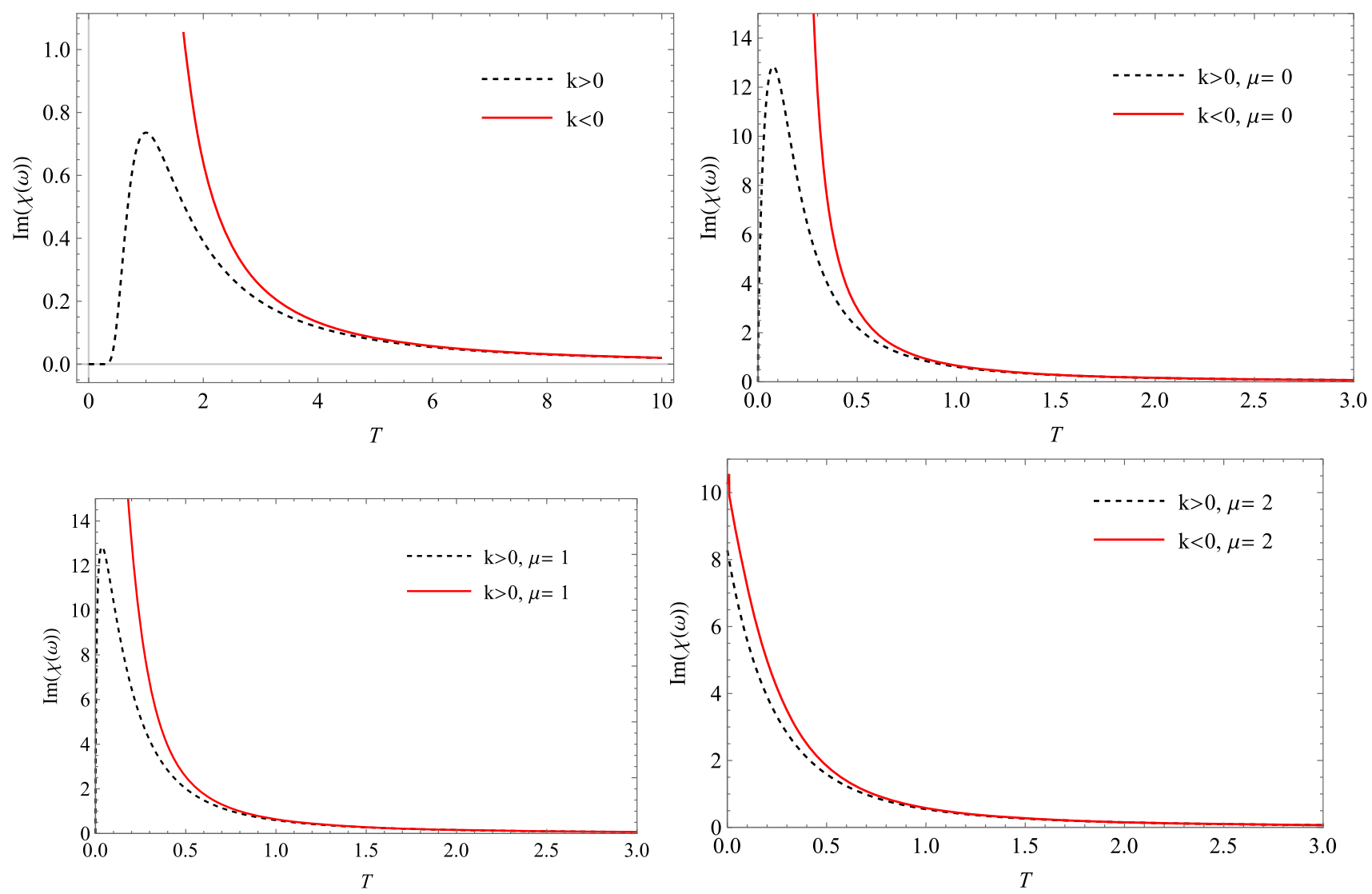

Fig. 3 Imaginary part of the admittance, as a function of the temperature $(T)$ for $k= \pm 0.18$, in various scenarios. Upper left panel: results from Ref. [54] where the backreaction contributions were not considered. Upper right panel: results from Ref. [55] where the backreaction

was considered although for zero chemical potential. Lower left and right panels: results achieved in this work, Eq. (45), considering both backreaction and finite chemical potential with $\mu=1$ and $\mu=2$, respectively

sis into two separate cases since the sign of the chemical potential will be crucial.

\subsubsection{Bosonic case}

Performing the integral, one gets

$s_{\mathrm{Breg}}^{2}(t)=\frac{2 \alpha^{\prime} e^{-\frac{k}{r_{h}^{2}}}}{r_{h}^{2}} \sum_{n=1}^{\infty} e^{\beta \mu n} \log \left(1+\frac{t^{2}}{n^{2} \beta^{2}}\right)$.

In order to calculate the mean square displacement given by Eq. (63), for the bosonic case, we take $\mu<0$, and consider the series expansion

$\frac{1}{e^{\beta(\omega-\mu)}-1}=\frac{e^{-\beta(\omega-\mu)}}{1-e^{-\beta(\omega-\mu)}}=\sum_{n=0}^{\infty} e^{-\beta(\omega-\mu)(n+1)}$.

Then, the bosonic regularized mean square displacement reads

$s_{\mathrm{Breg}}^{2}(t)=\frac{8 \alpha^{\prime} e^{-\frac{k}{r_{h}^{2}}}}{r_{h}^{2}} \sum_{n=1}^{\infty} \int_{0}^{\infty} \frac{d \omega}{\omega} e^{-\beta(\omega-\mu) n} \sin ^{2}\left(\frac{\omega t}{2}\right)$.

This expression can be rewritten as

$$
\begin{aligned}
s_{\mathrm{Breg}}^{2}(t)= & \frac{2 \alpha^{\prime} e^{-\frac{k}{r_{h}^{2}}}}{r_{h}^{2}}\left(2 \operatorname{Li}_{0}{ }^{(1,0)}\left(0, e^{\mu}\right)\right. \\
& -e^{\mu}\left(\Phi^{(0,1,0)}\left(e^{\mu}, 0,1+i \frac{t}{\beta}\right)\right. \\
& \left.\left.+\Phi^{(0,1,0)}\left(e^{\mu}, 0,1-i \frac{t}{\beta}\right)\right)\right),
\end{aligned}
$$

where $\mathrm{Li}_{n}^{(1,0)}(x, y)$ is the first derivative of the polylogarithm function of order $n$ with respect to its first argument $x$ and $\Phi^{(0,1,0)}(x, y, z)$ is the first derivative of the Lerch transcendent function with respect to the second argument $y$. 

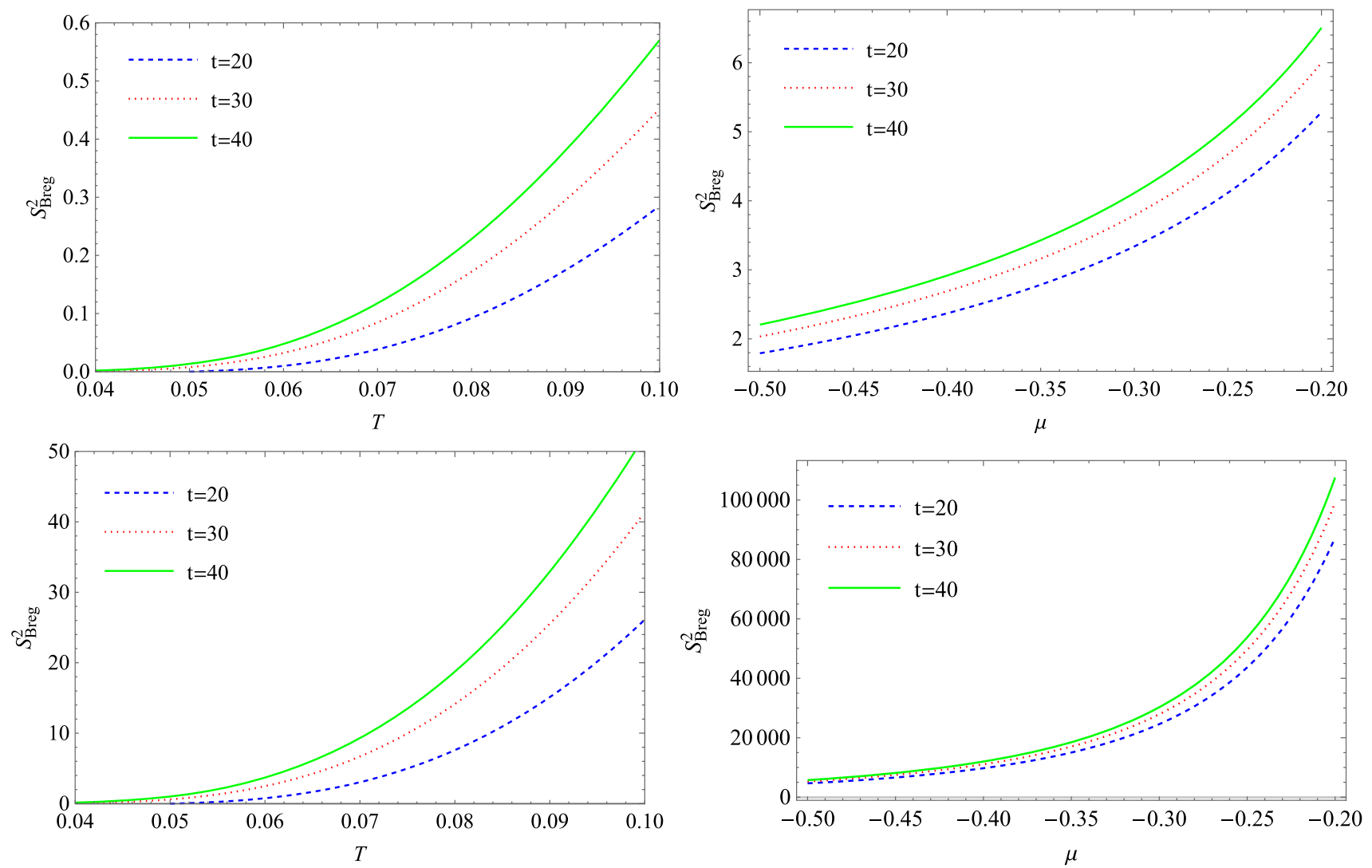

Fig. 4 Bosonic regularized mean square displacement $s_{\mathrm{Breg}}^{2}$ as a function of the temperature $T$ or the chemical potential $\mu$ for the late time approximation, Eq. (68), for some values of the time $t$. Upper panels: positive fixed value of $k$. Lower panels: negative fixed value of $k$

Now, it is interesting to consider the late time approximation $t \gg \beta$ for Eq. (66) as

$$
\begin{aligned}
s_{\text {Breg }}^{2}(t) \approx & \frac{2 \alpha^{\prime} e^{-\frac{k}{r_{h}^{2}}}}{r_{h}^{2}} \sum_{n=1}^{\infty} e^{\beta \mu n} \log \left(\frac{t^{2}}{n^{2} \beta^{2}}\right) \\
= & \frac{2 \alpha^{\prime} e^{-\frac{k}{r_{h}^{2}}}}{r_{h}^{2}}\left(-\sum_{n=1}^{\infty} e^{\beta \mu n} \log \left(n^{2}\right)\right. \\
& \left.+\sum_{n=1}^{\infty} e^{\beta \mu n} \log \left(\frac{t^{2}}{\beta^{2}}\right)\right) \\
= & \frac{2 \alpha^{\prime} e^{-\frac{k}{r_{h}^{2}}}}{r_{h}^{2}}\left(-\sum_{n=1}^{\infty} e^{\beta \mu n} \log \left(n^{2}\right)\right. \\
& \left.+\frac{2}{e^{-\beta \mu}-1} \log \left(\frac{t}{\beta}\right)\right),
\end{aligned}
$$

which converges since $\mu<0$. This result corresponds to a sub-diffusive regime as $s^{2} \sim \log t$, as seen in Ref. [99]. This behavior is shown in Fig. 4, for various values of the time $t$ against the temperature $T$ or the chemical potential $\mu$ for positive and negative $k$.
If one considers the limit $\mu / T \rightarrow 0$ in Eq. (66), one can rewrite that sum as

$$
\begin{aligned}
s_{\text {Breg }}^{2}(t) & =\frac{2 \alpha^{\prime} e^{-\frac{k}{r_{h}^{2}}}}{r_{h}^{2}} \sum_{n=1}^{\infty} e^{\beta \mu n} \log \left(1+\frac{t^{2}}{n^{2} \beta^{2}}\right) \\
& \approx \frac{2 \alpha^{\prime} e^{-\frac{k}{r_{h}^{2}}}}{r_{h}^{2}} \sum_{n=1}^{\infty} \log \left(1+\frac{t^{2}}{n^{2} \beta^{2}}\right) \\
& =\frac{2 \alpha^{\prime} e^{-\frac{k}{r_{h}^{2}}}}{r_{h}^{2}} \log \left(\frac{\beta \sinh \left(\frac{\pi t}{\beta}\right)}{\pi t}\right) .
\end{aligned}
$$

In this case, for long times, $t \gg \beta / \pi$, one has

$$
\begin{aligned}
s^{2} & =\frac{2 \alpha^{\prime} e^{-\frac{k}{r_{h}^{2}}}}{r_{h}^{2}}\left[\log \left(e^{\frac{\pi t}{\beta}}-e^{-\frac{\pi t}{\beta}}\right)-\log \left(\frac{2 \pi t}{\beta}\right)\right] \\
& \approx \frac{2 \pi \alpha^{\prime} e^{-\frac{k}{r_{h}^{2}}}}{\beta r_{h}^{2}} t .
\end{aligned}
$$

So, in the limit $\mu / T \rightarrow 0$, one recovers the usual linear behavior for the mean square displacement. 
On the other hand, for the short time approximation $t \ll \beta$, we find

$s_{\mathrm{Breg}}^{2}(t) \approx \frac{t^{2}}{\beta^{2}}\left(\sum_{n=1}^{\infty} \frac{e^{\beta \mu n}}{n^{2}}\right)=\frac{t^{2}}{\beta^{2}} \operatorname{Li}_{2}\left(e^{\beta \mu}\right)$,

where $\mathrm{Li}_{2}\left(e^{\beta \mu}\right)$ is the polylogarithm function of order 2 . As expected, this corresponds to the ballistic regime which goes like $s^{2} \sim t^{2}$, as shown in Fig. 5 .

\subsubsection{Fermionic case}

Here, we are going to consider the fermionic regularized mean square displacement. From Eq. (63), taking the Fermi Dirac distribution with $\mu>0$, we have

$s_{\text {Freg }}^{2}(t)=\frac{8 \alpha^{\prime} e^{-\frac{k}{r_{h}^{2}}}}{r_{h}^{2}} \int_{0}^{\infty} \frac{d \omega}{\omega}\left(\frac{\sin ^{2}\left(\frac{\omega t}{2}\right)}{e^{\beta(\omega-\mu)}+1}\right)$.

We can calculate this integral using the Sommerfeld expansion:

$$
\begin{aligned}
& \int_{0}^{\infty} \frac{d \omega}{\omega}\left(\frac{\sin ^{2}\left(\frac{\omega t}{2}\right)}{e^{\beta(\omega-\mu)}+1}\right) \\
& =\int_{0}^{\mu} \frac{\sin ^{2}\left(\frac{\omega t}{2}\right)}{\omega} d \omega+\left.\frac{\pi^{2}}{6 \beta^{2}} \frac{d}{d \omega}\left(\frac{\sin ^{2}\left(\frac{\omega t}{2}\right)}{\omega}\right)\right|_{\omega=\mu} \\
& =\frac{1}{2}(-\operatorname{Ci}(t \mu)+\log (t \mu)+\gamma) \\
& \quad+\frac{\pi^{2}}{6 \beta^{2}} \frac{\mu t \sin (\mu t)-2 \sin ^{2}\left(\frac{\mu t}{2}\right)}{2 \mu^{2}},
\end{aligned}
$$

where we kept terms up to order $1 / \mu^{2} \beta^{2}, \operatorname{Ci}(z)=-\int_{z}^{\infty} \frac{\cos (s)}{s}$ $d s$ is the Cosine integral function and $\gamma$ is Euler's constant, with numerical value $\gamma \approx 0.577216$.

In the low temperature regime, $\mu \beta=\frac{\mu}{T} \gg 1$, we can disregard the second term in the above equation and obtain for large times $t \gg \mu$

$s_{\text {Freg }}^{2}(t) \approx \frac{4 \alpha^{\prime} e^{-\frac{k}{r_{h}^{2}}}}{r_{h}^{2}}(\log (t \mu)+\gamma)$

which is again a sub-diffusive regime $s^{2} \sim \log t$, as in the bosonic case, or the ones seen in Ref. [99]. This behavior is shown in Fig. 6.

In order to take the limit $\mu / T \rightarrow 0$, here in the fermionic case, one needs to go back to Eq. (72), before the Sommerfeld expansion Eq. (73). Since this limit corresponds to a high temperature limit, one sees that the Fermi distribution goes over the classical one, where bosons and fermions are degenerate. In this case the the mean square displacement goes as $s^{2} \sim t$, as expected $[54,55]$.
On the other hand, for small times, $t \ll \mu$, the mean square displacement can be approximated as

$s_{\text {Freg }}^{2}(t) \approx \frac{\alpha^{\prime} e^{-\frac{k}{r_{h}^{2}}}}{r_{h}^{2}}\left(\frac{\pi^{2}}{3 \beta^{2}}+\mu^{2}\right) t^{2}$,

which corresponds to a usual ballistic regime $s^{2} \sim t^{2}$. From Eq. (75) one can see its behavior in Fig. 7.

It is interesting to note that for the bosonic and fermionic cases, Eqs. (68) and (74), both have as a large time approximation a sub-diffusive behavior with $s^{2} \sim \log (t)$. Such a logarithm time dependence was found in ultraslow scaled Brownian motion representing physical systems in Sinai diffusion. For instance, in quenched random energy landscapes, random walks on bundled structures, colloidal hard sphere systems at the liquid-glass transition, or granular gases in the homogeneous cooling stage, as discussed in Ref. [99].

This is a slower process than the usual $s^{2} \sim t$, found for the Brownian motion that follows the Langevin equation

$m \ddot{x}=-\gamma m \dot{x}+R(t)$,

where $\gamma$ is the drag coefficient, $m$ is the mass and $R(t)$ is an stochastic variable representing the random force acting on the particle.

\section{Checking the fluctuation-dissipation theorem for bosonic and fermionic cases}

Now, after calculating the admittance and the correlation functions for the bosonic and fermionic systems, we are ready to check the fluctuation-dissipation theorem in both cases, as stated in Sect. 2.

First of all, we can rewrite the r.h.s of Eqs. (4) and (5) as

$$
\begin{aligned}
& \mathcal{F}^{-1}\left[\left(1+2 n_{B}\right) \Im \chi(\omega)\right] \\
& =\frac{1}{2 \pi} \int_{-\infty}^{\infty} d \omega\left(1+\frac{2}{e^{\beta(|\omega|-\mu)}-1}\right) \Im \chi(|\omega|) e^{i \omega t}, \\
& \mathcal{F}^{-1}\left[\left(1+2 n_{F}\right) \Im \chi(\omega)\right] \\
& =\frac{1}{2 \pi} \int_{-\infty}^{\infty} d \omega\left(1+\frac{2}{e^{\beta(|\omega|-\mu)}+1}\right) \Im \chi(|\omega|) e^{i \omega t},
\end{aligned}
$$

where we are considering the frequencies as positive in the physical quantities. From the admittance, Eq. (43), the above equations can be calculated as

$$
\begin{aligned}
& \frac{1}{2 \pi} \int_{-\infty}^{\infty} d \omega\left(1+\frac{2}{e^{\beta(|\omega|-\mu)} \mp 1}\right) \Im \chi(|\omega|) e^{i \omega t} \\
& =\frac{\alpha^{\prime} e^{-\frac{k}{r_{h}^{2}}}}{r_{h}^{2}} \int_{-\infty}^{\infty} \frac{d \omega}{|\omega|}\left(1+\frac{2}{e^{\beta(|\omega|-\mu)} \mp 1}\right) e^{i \omega t},
\end{aligned}
$$

where the minus (plus) sign represents bosons (fermions), 

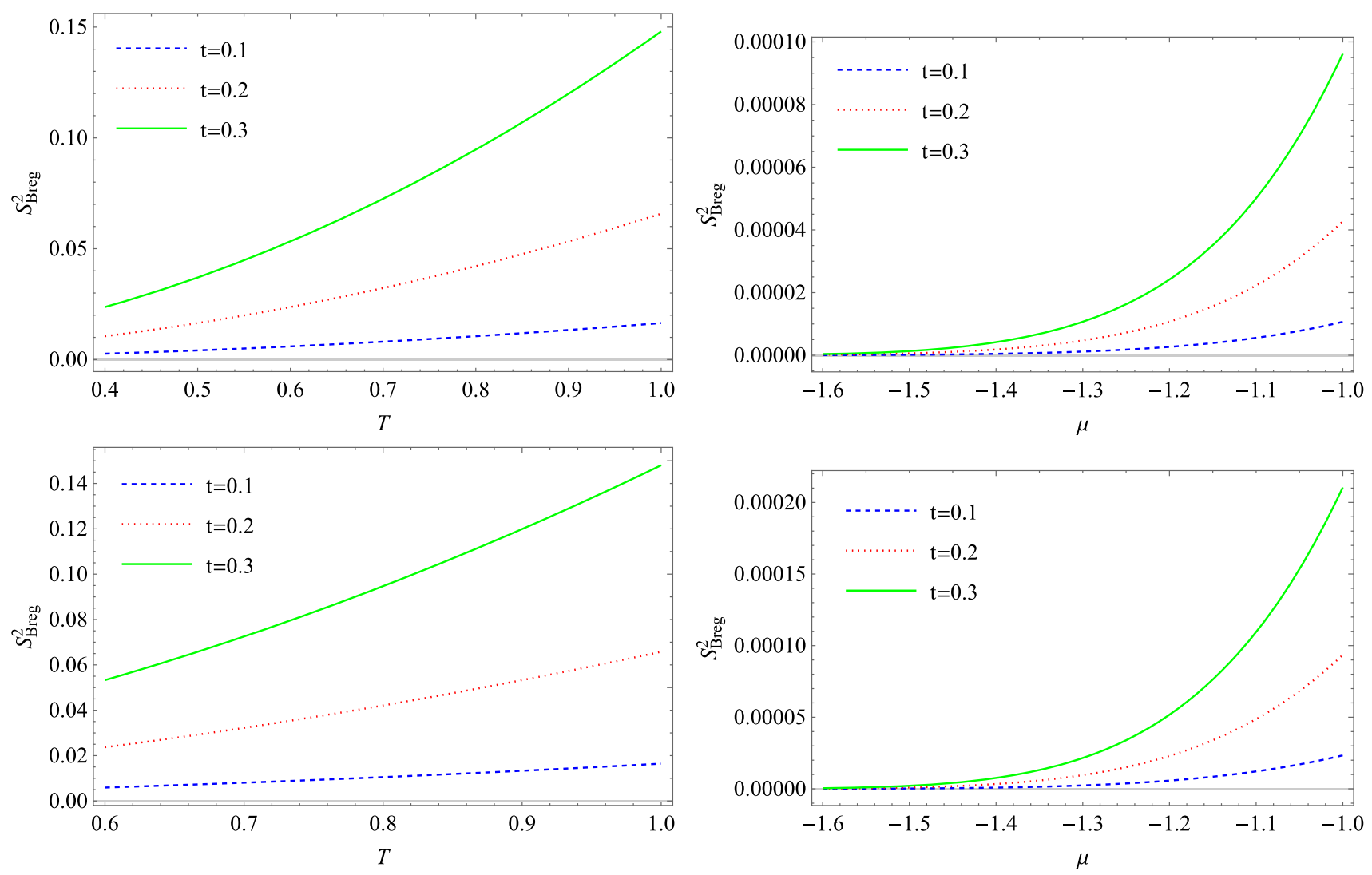

Fig. 5 Bosonic regularized mean square displacement $s_{\text {Breg }}^{2}$ as a function of the temperature $T$ or the chemical potential $\mu$ for the short time approximation, Eq. (71), for some values of the time $t$. Upper panels: positive fixed value of $k$. Lower panels: negative fixed value of $k$

On the other hand, from the bosonic and fermionic correlation functions, Eqs. (59) and (60), the 1.h.s. of Eqs. (4) and (5) become

$$
\begin{aligned}
G_{\mathrm{Sym}}(t) & =\frac{\alpha^{\prime} e^{-\frac{k}{r_{h}^{2}}}}{r_{h}^{2}} \int_{0}^{\infty} \frac{d \omega}{\omega}\left(\frac{4 \cos (\omega t)}{e^{\beta(\omega-\mu)} \mp 1}+e^{-i \omega t}+e^{i \omega t}\right) \\
& =\frac{\alpha^{\prime} e^{-\frac{k}{r_{h}^{2}}}}{r_{h}^{2}} \int_{0}^{\infty} \frac{d \omega}{|\omega|}\left(\frac{2\left(e^{-i \omega t}+e^{i \omega t}\right)}{e^{\beta(|\omega|-\mu)} \mp 1}+e^{-i \omega t}+e^{i \omega t}\right),
\end{aligned}
$$

where again the minus (plus) sign represents bosons (fermions). Noting that

$$
\begin{aligned}
& \int_{0}^{\infty} d \omega f(|\omega|) e^{i \omega t}+\int_{0}^{\infty} d \omega f(|\omega|) e^{-i \omega t} \\
& =\int_{-\infty}^{\infty} d \omega f(|\omega|) e^{i \omega t}
\end{aligned}
$$

we finally have that the symmetric Green's function given by Eq. (80) can be rewritten as

$$
G_{\text {Sym }}=\frac{\alpha^{\prime} e^{-\frac{k}{r_{h}^{2}}}}{r_{h}^{2}} \int_{-\infty}^{\infty} \frac{d \omega}{|\omega|}\left(\frac{2}{e^{\beta(|\omega|-\mu)} \mp 1}+1\right) e^{i \omega t},
$$

which coincides with Eq. (79). Then, we checked the Eqs. (4) and (5), completing our verification of the fluctuationdissipation theorem in the holographic string/gauge deformed metric with backreaction at finite temperature and chemical potential.

\section{Conclusions}

In this work we investigated the effects of a conformal exponential deformation and its backreaction in an AdS-RN spacetime. Then using the AdS/CFT dictionary we studied the fluctuation and dissipation of a test particle in a thermal bath with finite chemical potential. Solving the EMD field equations we obtain the backreacted horizon function $f(r)$ depending on the chemical potential $\mu$ and the deformation parameter $k$. From this horizon function we obtain the Hawking temperature which is also the temperature of the dual field theory, as a function of the horizon radius $r_{h}$, as can be seen in Fig. 2. From the Nambu-Goto action we obtain the equation of motion for the probe string in the metric considered. The solutions of this equation in different regions are obtained from a monodromy patch procedure. Then, we calculate the admittance or linear response, and the friction coefficient $\gamma$. 

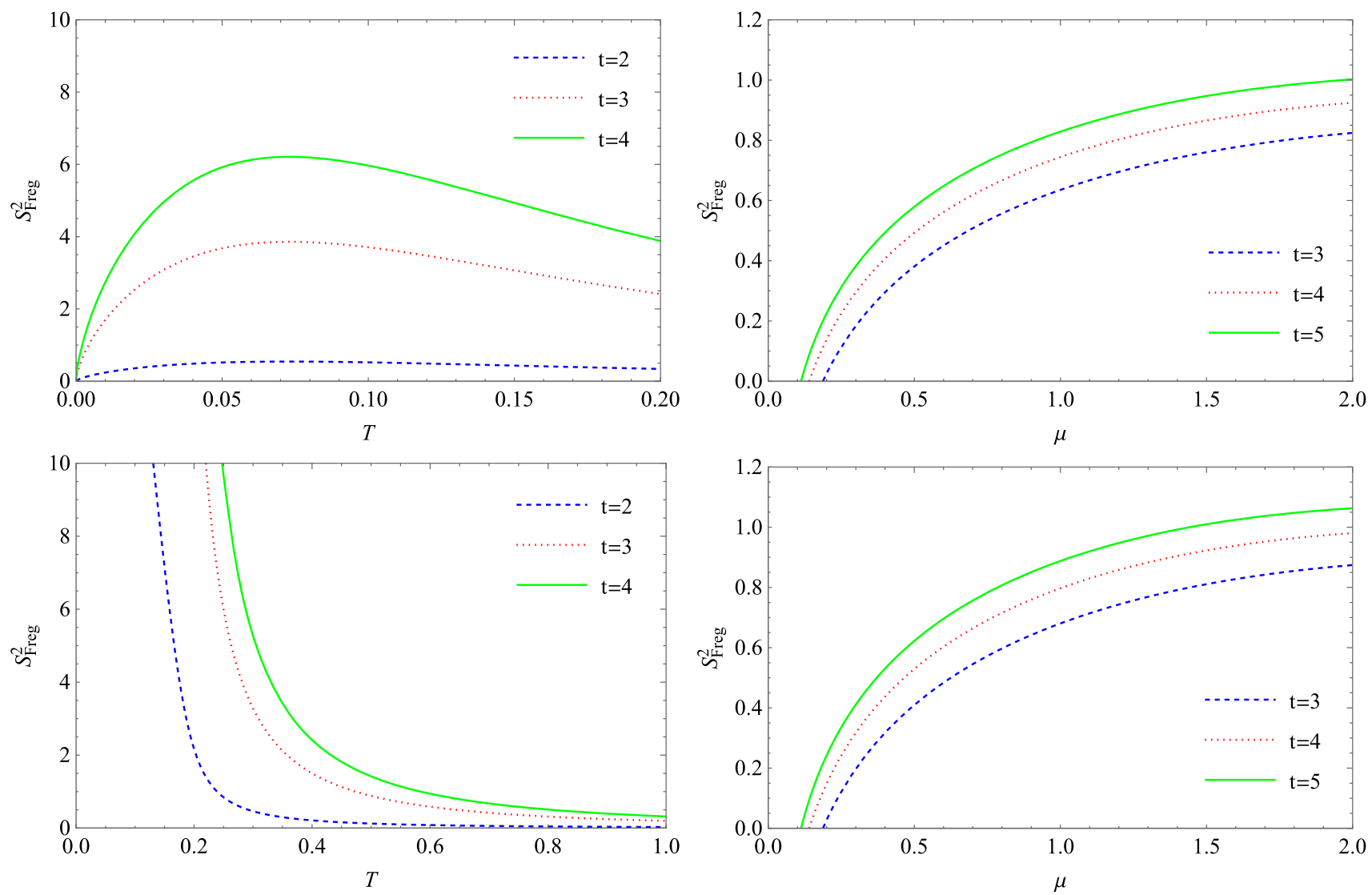

Fig. 6 Fermionic regularized mean square displacement $s_{\text {Freg }}^{2}$ as a function of the temperature $T$ or the chemical potential $\mu$ for the large time approximation, Eq. (74), for some values of the time $t$. Upper panels: positive fixed value of $k$. Lower panels: negative fixed value of $k$

An important contribution of this work is given by the admittance calculated in Eq. (45), which depends on the deformed metric with backreaction at finite temperature and chemical potential. The temperature dependence of this quantity is a non-trivial one. Figure 3 summarizes this result in comparison with others discussed previously in the literature. Note that these other models can be understood as particular cases of the one presented here.

Another important result obtained here is the diffusion of the particle represented by the string endpoint. It was analyzed considering bosonic and fermionic statistical distributions. In the two cases we observed that the mean square displacement behaves as $s^{2} \sim \log t$ for large times indicating a sub-diffusive regime. Such a behavior was found in ultra-slow scaled Brownian motion, known as Sinai diffusion. Note that Ref. [99] reports this diffusion process with many physical realizations.

Therefore the sub-diffusive regime obtained here differs, for instance, from the one of a Brownian motion studied as a dual for the AdS-BTZ black hole presented in Ref. [40]. We interpret this difference due to the presence of a chemical potential in our set up. This assumption is supported by the result presented in Ref. [55]. There, the authors considered a similar gravitational background in absence of a chemical potential and achieved the typical diffusive behavior $s^{2} \sim t$. We recovered this result in Sect. 4.3 for bosons and fermions in the limit $\mu / T \rightarrow 0$, in the large time regime.

In the last section we checked the fluctuation dissipation theorem, for both fermionic and bosonic cases: $\frac{1}{2}(\langle x(t) x(0)\rangle+\langle x(0) x(t)\rangle)=\mathcal{F}^{-1}\left[\left(1+2 n_{B / F}\right) \Im \chi(\omega)\right]$. This was accomplished from the admittance and the correlation functions calculated in Sects. 4.2 and 4.3, respectively.

Acknowledgements The authors would like to thank Alfonso Ballon Bayona for discussions. N.G.C. is supported by Conselho Nacional de Desenvolvimento Científico e Tecnológico (CNPq). H.B.-F. and C.A.D.Z. are partially supported by Conselho Nacional de Desenvolvimento Científico e Tecnológico (CNPq) under the grants Nos. 311079/2019-9 and 309982/2018-9, respectively. C.A.D.Z is also partially supported by Fundação Carlos Chagas Filho de Amparo à Pesquisa do Estado do Rio de Janeiro - FAPERJ under grant E-26/201.447/2021. This work is also supported by Coordenação de Aperfeiçoamento de Pessoal de NÃvel Superior (CAPES), under finance code 001.

Data Availability Statement This manuscript has no associated data or the data will not be deposited. [Authors' comment: The results in this paper are obtained analytically, hence, it does not use any data.]

Open Access This article is licensed under a Creative Commons Attribution 4.0 International License, which permits use, sharing, adaptation, 

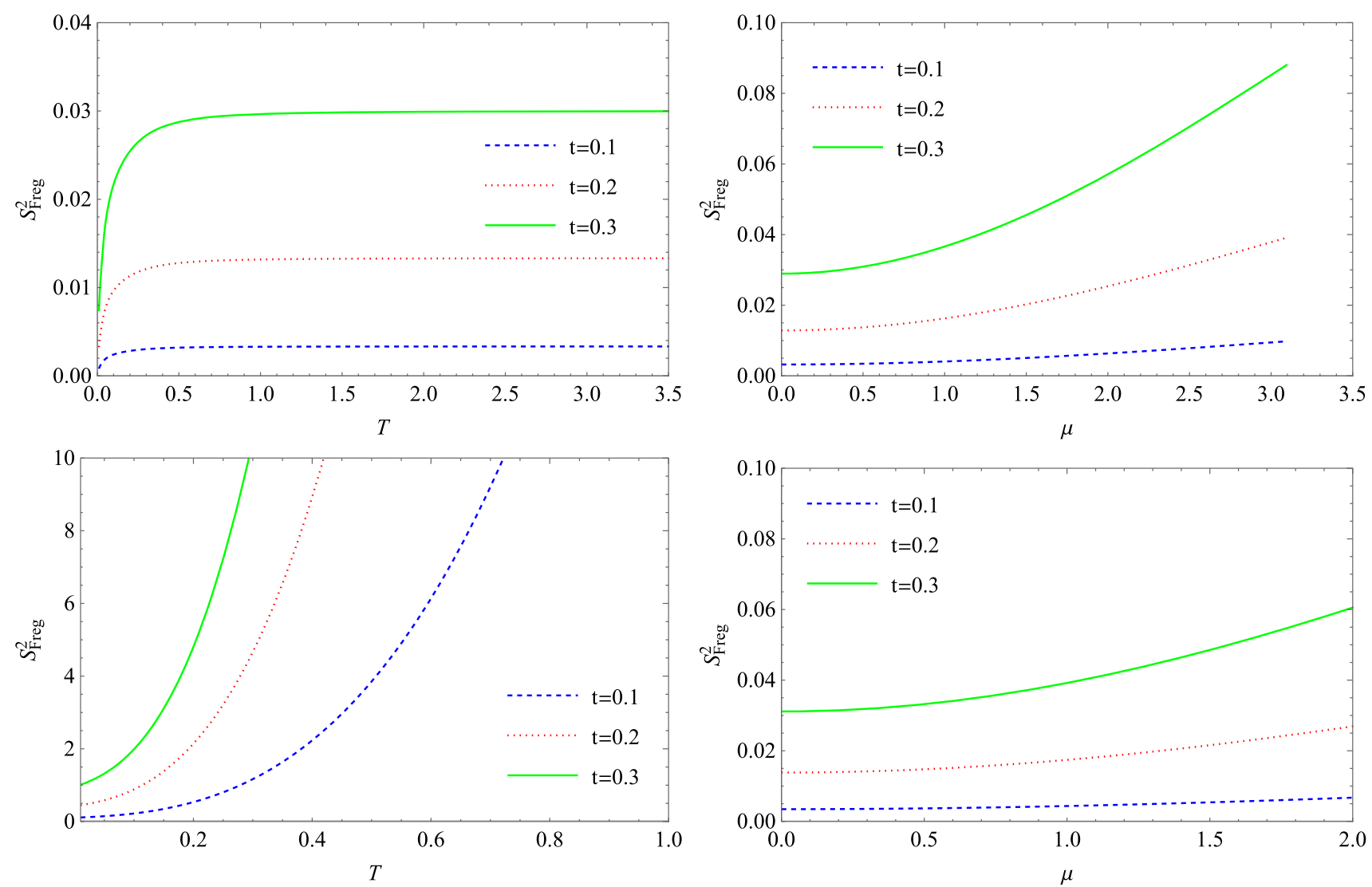

Fig. 7 Fermionic regularized mean square displacement $s_{\text {Freg }}^{2}$ as a function of the temperature $T$ or the chemical potential $\mu$ for the short time approximation, Eq. (75), for some values of the time $t$. Upper panels: positive fixed value of $k$. Lower panels: negative fixed value of $k$

distribution and reproduction in any medium or format, as long as you give appropriate credit to the original author(s) and the source, provide a link to the Creative Commons licence, and indicate if changes were made. The images or other third party material in this article are included in the article's Creative Commons licence, unless indicated otherwise in a credit line to the material. If material is not included in the article's Creative Commons licence and your intended use is not permitted by statutory regulation or exceeds the permitted use, you will need to obtain permission directly from the copyright holder. To view a copy of this licence, visit http://creativecomm ons.org/licenses/by/4.0/.

Funded by SCOAP ${ }^{3}$.

\section{Appendix A: Monodromy patch}

Here in this Appendix we will find approximate analytical solution for the Schrödinger-like Eq. (36) in three different regions in the bulk of the deformed AdS space.

\section{A.1 Region A: $\omega^{2} \gg V(r)$}

The first region, dubbed as $\mathbf{A}$, is nearby the event horizon, i.e. $r \approx r_{h}$. In this case, $V(r) \ll \omega^{2}$, and the Schrödinger-like equation reads $\frac{d^{2} \psi\left(r_{*}\right)}{d r_{*}^{2}}+\omega^{2} \psi\left(r_{*}\right)=0$

which has the ingoing solution

$\psi\left(r_{*}\right)=A_{1} e^{-i \omega r_{*}}$

Close to the horizon $\left(r \approx r_{h}\right)$, we can assume that for low frequencies we have $\omega r_{*} \ll 1$. Then one can expand Eq. (A2) as:

$\psi\left(r_{*}\right)=A_{1}-i A_{1} \omega r_{*}$.

Using this equation and the Bogoliubov transformation, $B(r)=-k /\left(2 r^{2}\right)-\log (r)$, we can compute $h_{\omega}\left(r_{*}\right)$ in this region as:

$h_{\omega}^{A}\left(r_{*}\right)=\frac{e^{-\frac{k}{2 r_{h}^{2}}}}{r_{h}}\left(A_{1}-i \omega A_{1} r_{*}\right)$.

Close to the horizon, the tortoise coordinate $r_{*}$ is given by

$\int^{r} \frac{1}{r_{h}^{2} f^{\prime}\left(r_{h}\right)\left(r^{\prime}-r_{h}\right)} d r^{\prime}=\frac{1}{r_{h}^{2} f^{\prime}\left(r_{h}\right)} \log \left(\frac{r}{r_{h}}-1\right)$. 
Then, Eq. (A4) gives the expression for $h_{\omega}^{a}\left(r_{*}\right)$

$h_{\omega}^{A}\left(r_{*}\right)=A_{1} \frac{e^{-\frac{k}{2 r_{h}^{2}}}}{r_{h}}\left(1-i \frac{\omega}{r_{h}^{2} f^{\prime}\left(r_{h}\right)} \log \left(\frac{r}{r_{h}}-1\right)\right)$.

\section{A. 2 Region B: $V(r) \gg \omega^{2}$}

Now, we analyze the region where $V(r) \gg \omega^{2}$. First, note that the potential term in Eq. (36) comes from the term without $\omega^{2}$ in Eq. (34). Then, in this second region we can drop this last term and work with (34) in the form

$\frac{d}{d r}\left(r^{4} f(r) e^{\frac{k}{r^{2}}} h_{\omega}^{\prime}\right)=0$.

Solving this equation we obtain

$h_{\omega}^{B}(r)=\int^{r} \frac{B_{1}}{r^{\prime 4} f\left(r^{\prime}\right) e^{\frac{k}{r^{2}}}} d r^{\prime}+B_{2}$.

In the IR regime, we can approximate the integral above using the fact that $f(r)$ has a simple pole at the horizon. Then we can write

$h_{\omega(\mathrm{IR})}^{B}(r)=\frac{B_{1}}{e^{\frac{k}{r_{h}^{2}}} r_{h}^{4} f^{\prime}\left(r_{h}\right)} \log \left(\frac{r}{r_{h}}-1\right)+B_{2}$.

Comparing the last expression with (A6) we have

$B_{1}=-i A_{1} r_{h} \omega e^{\frac{k}{2 r_{h}^{2}}}$,

$B_{2}=A_{1} \frac{e^{-\frac{k}{2 r_{h}^{2}}}}{r_{h}}$.

For the UV regime, $f(r) \approx 1$ and the solution becomes

$h_{\omega(\mathrm{UV})}^{B}(r)=\int^{r} \frac{B_{1}}{r^{\prime 4} e^{\frac{k}{r^{\prime 2}}}} d r^{\prime}+B_{2}$.

After integrating we get large values of $r$

$h_{\omega(\mathrm{UV})}^{B}(r) \approx-\frac{B_{1}}{3 r^{3}}+B_{2}$.

\section{A.3 Region C: the deep UV}

In the deep UV region we have that the horizon function becomes close to the unity and we must solve the equation of motion in the form

$\frac{d}{d r}\left(r^{4} e^{\frac{k}{r^{2}}} h_{\omega}^{\prime}\right)+\omega^{2} e^{\frac{k}{r^{2}}} h_{\omega}=0$,

whose general solution is

$$
\begin{aligned}
h_{\omega}^{C}(r)= & C_{1{ }_{1} F_{1}}\left(\frac{\omega^{2}}{4 k},-\frac{1}{2},-\frac{k}{r^{2}}\right) \\
& +C_{2} \frac{k^{3 / 2}}{r^{3}} F_{1}\left(\frac{3}{2}+\frac{\omega^{2}}{4 k}, \frac{5}{2},-\frac{k}{r^{2}}\right) .
\end{aligned}
$$

Close to the boundary this solution can be expanded as a power series in $1 / r$

$h_{\omega}^{C}(r)=C_{1}+\frac{C_{1} \omega^{2}}{2 r^{2}}+\frac{C_{2} k^{3 / 2}}{r^{3}}+O\left(\left(\frac{1}{r}\right)^{4}\right)$.

Keeping only terms until $O(\omega)$,we have:

$h_{\omega}^{C}(r) \approx C_{1}+\frac{C_{2} k^{3 / 2}}{r^{3}}$.

Matching this result with Eq. (A12), we find

$C_{1}=B_{2}=A_{1} \frac{e^{-\frac{k}{2 r_{h}^{2}}}}{r_{h}}, \quad C_{2}=-\frac{B_{1}}{3 k^{3 / 2}}=\frac{i A_{1} r_{h} \omega e^{\frac{k}{2 r_{h}^{2}}}}{3 k^{3 / 2}}$

Now we can write the solution close to the boundary as

$h_{\omega}^{C}(r) \approx A_{1}\left(\frac{e^{-\frac{k}{2 r_{h}^{2}}}}{r_{h}}+\frac{i \omega r_{h} e^{\frac{k}{2 r_{h}^{2}}}}{3 r^{3}}\right)$.

\section{Appendix B: Normalization}

The ingoing modes were calculated in the previous section. The outgoing modes come from the ingoing modes given by Eq. (A2). Then, the outgoing modes near the horizon read:

$\psi^{\text {out }}(r)=A_{2} e^{i \omega r_{*}}$.

At the boundary, analogously to Eq. (38), the outgoing solutions are

$h_{\omega}^{\text {out }}(r) \approx\left(\frac{e^{-\frac{k}{2 r_{h}^{2}}}}{r_{h}}-\frac{i \omega r_{h} e^{\frac{k}{2 r_{h}^{2}}}}{3 r^{3}}\right)$.

Thus, for large $r$ and small $\omega$ the general solution for the equation of motion can be written as

$$
\begin{aligned}
h_{\omega}^{\text {bound }}(r)= & A \frac{e^{-\frac{k}{2 r_{h}^{2}}}}{r_{h}}\left[\left(1+\frac{i \omega r_{h}^{2} e^{\frac{k}{r_{h}^{2}}}}{3 r^{3}}\right)\right. \\
& \left.+B\left(1-\frac{i \omega r_{h}^{2} e^{\frac{k}{r_{h}^{2}}}}{3 r^{3}}\right)\right] .
\end{aligned}
$$

Near the boundary we also can write a general solution yet as

$$
\begin{aligned}
h_{\omega} & =A\left[h_{\omega}^{\text {out }}(r)+B h_{\omega}^{\text {in }}(r)\right] \\
& =A\left[{ }_{1} F_{1}\left(\frac{\omega^{2}}{4 k} ;-\frac{1}{2} ;-\frac{k}{r^{2}}\right)\right.
\end{aligned}
$$




$$
\begin{aligned}
& -\frac{i r_{h}^{2} \omega e^{\frac{k}{r_{h}^{2}}}{ }_{1} F_{1}\left(\frac{\omega^{2}}{4 k}+\frac{3}{2} ; \frac{5}{2} ;-\frac{k}{r^{2}}\right)}{3 r^{3}} \\
& B\left({ }_{1} F_{1}\left(\frac{\omega^{2}}{4 k} ;-\frac{1}{2} ;-\frac{k}{r^{2}}\right)\right. \\
& \left.\left.+\frac{i r_{h}^{2} \omega e^{\frac{k}{r_{h}^{2}}}{ }_{1} F_{1}\left(\frac{\omega^{2}}{4 k}+\frac{3}{2} ; \frac{5}{2} ;-\frac{k}{r^{2}}\right)}{3 r^{3}}\right)\right] .
\end{aligned}
$$

Imposing Neumann boundary condition on the brane at $r=$ $r_{b}$ we can write

$B=-\frac{\Xi}{\Xi^{*}}$,

which is a pure phase, and we define

$$
\begin{aligned}
\Xi= & i r_{h}^{2} e^{\frac{k}{r_{h}^{2}}}\left[\left(6 k+\omega^{2}\right){ }_{1} F_{1}\left(\frac{\omega^{2}}{4 k}+\frac{5}{2} ; \frac{7}{2} ;-\frac{k}{r_{b}^{2}}\right)\right. \\
& \left.-15 r_{b 1}^{2} F_{1}\left(\frac{1}{4}\left(\frac{\omega^{2}}{k}+6\right) ; \frac{5}{2} ;-\frac{k}{r_{b}^{2}}\right)\right] \\
& +15 r_{b}^{3} \omega_{1} F_{1}\left(\frac{\omega^{2}}{4 k}+1 ; \frac{1}{2} ;-\frac{k}{r_{b}^{2}}\right) .
\end{aligned}
$$

On the other side, close to the horizon we can write the general solution as

$h_{\omega}^{\text {hor }}(r)=A \frac{e^{-\frac{k}{2 r^{2}}}}{r}\left[e^{-i \frac{\omega \log \left(\frac{r}{r_{h}}-1\right)}{r_{h}^{2} f^{\prime}\left(r_{h}\right)}}+B e^{i \frac{\omega \log \left(\frac{r}{r_{h}}-1\right)}{r_{h}^{2} f^{\prime}\left(r_{h}\right)}}\right]$,

and following [40], we impose an extra Neumann boundary condition at $\frac{r}{r_{h}}=1+\epsilon, \epsilon \ll 1$. So, we have

$B=-\left.\frac{h_{\omega}^{\prime \text { in }}(r)}{h_{\omega}^{\prime \text { out }}(r)}\right|_{\frac{r}{r_{h}}=1+\epsilon} \approx e^{-\frac{2 i \omega}{r_{h}^{2} f^{\prime}\left(r_{h}\right)} \log \left(\frac{1}{\epsilon}\right)}$.

Matching this equation with (B5) one finds that the frequencies are discrete

$\Delta \omega=\frac{\pi r_{h}^{2} f^{\prime}\left(r_{h}\right)}{\log \left(\frac{1}{\epsilon}\right)}=\frac{4 \pi^{2} T}{\log \left(\frac{1}{\epsilon}\right)}$.

From the Klein-Gordon inner product we can normalize the solutions as $[40,53,54,98]$

$\left(X_{\omega}, X_{\omega}\right)=\frac{\omega}{\pi \alpha^{\prime}} \int_{r_{h}}^{r_{b}} d r \frac{e^{\frac{k}{r^{2}}}}{f(r)}\left|h_{\omega}(r)\right|^{2}=1$.

This integral is dominated by the near horizon contribution. First, in this region, at order $\omega$, we have

$$
\begin{aligned}
\left|h_{\omega}(r)\right|^{2} & =2\left|h_{\omega}^{\text {out }}(r)\right|^{2}+2 \Re\left(\left(h_{\omega}^{\text {out }}(r)\right)^{2} B^{*}\right) \\
& \approx 2|A|^{2} \frac{e^{-\frac{k}{r^{2}}}}{r^{2}}
\end{aligned}
$$

$$
\left\{1+\Re\left[B^{*}\left(1+\frac{2 i \omega \log \left(\frac{r}{r_{h}}-1\right)}{r_{h}^{2} f^{\prime}\left(r_{h}\right)}\right)\right]\right\}
$$

where $\Re$ represents the real part of the argument. Second, from Eq. (B5), up to order $\omega$, we can write that

$B^{*}=1-\frac{r_{b} \omega\left(2 i e^{-\frac{k}{r_{h}^{2}}}\right)}{r_{h}^{2}}+O\left(\frac{\sqrt{|k|}}{r_{b}}\right)$.

Note that the limit $\omega \rightarrow 0$ should be taken before the limit $r \rightarrow \infty$.

Then, using these results and performing the integral (B10), we get in leading order (in $r_{b}$ and $\omega$ ) close to the horizon at $r=r_{h}+\epsilon r_{h}$

$$
\begin{aligned}
& \frac{4 \omega|A|^{2}}{\pi \alpha^{\prime}} \int_{r_{h}+\epsilon r_{h}} \frac{d r^{\prime}}{r^{2} f(r)} \approx \frac{4 \omega|A|^{2}}{\pi \alpha^{\prime}} \int_{r_{h}+\epsilon r_{h}} \frac{d r^{\prime}}{r_{h}^{2} f^{\prime}\left(r_{h}\right)\left(r^{\prime}-r_{h}\right)} \\
& =\frac{4 \omega|A|^{2}}{\pi \alpha^{\prime} r_{h}^{2} f^{\prime}\left(r_{h}\right)} \log \left(\frac{1}{\epsilon}\right) .
\end{aligned}
$$

Thus, by imposing the normalization given by Eq. (B10), we obtain

$A=\sqrt{\frac{\pi \alpha^{\prime} r_{h}^{2} f^{\prime}\left(r_{h}\right)}{4 \omega \log \left(\frac{1}{\epsilon}\right)}}$.

\section{References}

1. R. Kubo, The fluctuation-dissipation theorem. Rep. Prog. Phys. 29, 255 (1966)

2. R. Kubo, M. Toda, N. Hashitsume, Statistical physics II: nonequilibrium statistical mechanics, vol. 2 (Springer Science \& Business Media, Berlin, 1991)

3. J.C. Collins, M.J. Perry, Superdense matter: neutrons or asymptotically free quarks? Phys. Rev. Lett. 34, 1353 (1975)

4. N. Cabibbo, G. Parisi, Exponential hadronic spectrum and quark liberation. Phys. Lett. B 59, 67-69 (1975)

5. E. Shuryak, Strongly coupled quark-gluon plasma in heavy ion collisions. Rev. Mod. Phys. 89, 035001 (2017). arXiv:1412.8393 [hep-ph]

6. I. Arsene et al. [BRAHMS], Quark gluon plasma and color glass condensate at RHIC? The Perspective from the BRAHMS experiment. Nucl. Phys. A 757, 1-27 (2005). arXiv:nucl-ex/0410020

7. B.B. Back et al. [PHOBOS], The PHOBOS perspective on discoveries at RHIC. Nucl. Phys. A 757, 28-101 (2005). arXiv:nucl-ex/0410022

8. J. Adams et al. [STAR], Experimental and theoretical challenges in the search for the quark gluon plasma: the STAR Collaboration's critical assessment of the evidence from RHIC collisions. Nucl. Phys. A 757, 102-183 (2005). arXiv:nucl-ex/0501009

9. K. Adcox et al. [PHENIX], Formation of dense partonic matter in relativistic nucleus-nucleus collisions at RHIC: experimental evaluation by the PHENIX collaboration. Nucl. Phys. A 757, 184283 (2005). arXiv:nucl-ex/0410003 
10. K. Aamodt et al. [ALICE], The ALICE experiment at the CERN LHC. JINST 3, S08002 (2008)

11. K. Aamodt et al. [ALICE], Phys. Rev. Lett. 106, 032301 (2011). arXiv:1012.1657 [nucl-ex]

12. G.D. Moore, N. Schlusser, The nonperturbative contribution to asymptotic masses. arXiv:2009.06614 [hep-lat]

13. M. Bluhm, A. Kalweit, M. Nahrgang, M. Arslandok, P. BraunMunzinger, S. Floerchinger, E.S. Fraga, M. Gazdzicki, C. Hartnack, C. Herold et al., Dynamics of critical fluctuations: theoryphenomenology—heavy-ion collisions. Nucl. Phys. A 1003, 122016 (2020). arXiv:2001.08831 [nucl-th]

14. G. Kadam, H. Mishra, M. Panero, Critical exponents and transport properties near the QCD critical endpoint from the statistical bootstrap model. arXiv:2011.02171 [hep-ph]

15. V.A. Goy, V. Bornyakov, D. Boyda, A. Molochkov, A. Nakamura, A. Nikolaev, V. Zakharov, Sign problem in finite density lattice QCD. PTEP 2017(3), $031 D 01$ (2017). arXiv:1611.08093 [hep-lat]

16. M. Troyer, U.J. Wiese, Computational complexity and fundamental limitations to fermionic quantum Monte Carlo simulations. Phys. Rev. Lett. 94, 170201 (2005). arXiv:cond-mat/0408370

17. S. Borsanyi, Z. Fodor, J.N. Guenther, R. Kara, S.D. Katz, P. Parotto, A. Pasztor, C. Ratti, K.K. Szabo, The QCD crossover at finite chemical potential from lattice simulations. Phys. Rev. Lett. 125(5), 052001 (2020). arXiv:2002.02821 [hep-lat]

18. O. Aharony, S.S. Gubser, J.M. Maldacena, H. Ooguri, Y. Oz, Large $\mathrm{N}$ field theories, string theory and gravity. Phys. Rep. 323, 183-386 (2000). arXiv:hep-th/9905111

19. G. Policastro, D.T. Son, A.O. Starinets, The shear viscosity of strongly coupled $\mathrm{N}=4$ supersymmetric Yang-Mills plasma. Phys. Rev. Lett. 87, 081601 (2001). arXiv:hep-th/0104066

20. Y. Kim, C.H. Lee, H.U. Yee, Holographic nuclear matter in AdS/QCD. Phys. Rev. D 77, 085030 (2008). arXiv:0707.2637 [hep$\mathrm{ph}]$

21. M. Panero, Thermodynamics of the QCD plasma and the large-N limit. Phys. Rev. Lett. 103, 232001 (2009). arXiv:0907.3719 [heplat]

22. O. DeWolfe, S.S. Gubser, C. Rosen, D. Teaney, Heavy ions and string theory. Prog. Part. Nucl. Phys. 75, 86-132 (2014). arXiv:1304.7794 [hep-th]

23. J. Casalderrey-Solana, H. Liu, D. Mateos, K. Rajagopal, U.A. Wiedemann, Gauge/string duality, hot QCD and heavy ion collisions. arXiv: 1101.0618 [hep-th]

24. I.Y. Aref'eva, K. Rannu, P. Slepov, Holographic anisotropic model for heavy quarks in anisotropic hot dense QGP with external magnetic field. arXiv:2011.07023 [hep-th]

25. P. Colangelo, F. Giannuzzi, S. Nicotri, Holography, heavy-quark free energy, and the QCD phase diagram. Phys. Rev. D 83, 035015 (2011). arXiv:1008.3116 [hep-ph]

26. D. Li, M. Huang, Q.S. Yan, A dynamical soft-wall holographic QCD model for chiral symmetry breaking and linear confinement. Eur. Phys. J. C 73, 2615 (2013). arXiv:1206.2824 [hep-th]

27. H. Bohra, D. Dudal, A. Hajilou, S. Mahapatra, Anisotropic string tensions and inversely magnetic catalyzed deconfinement from a dynamical AdS/QCD model. Phys. Lett. B 801, 135184 (2020). arXiv:1907.01852 [hep-th]

28. K. Ghoroku, K. Kashiwa, Y. Nakano, M. Tachibana, F. Toyoda, Extension to imaginary chemical potential in a holographic model. Phys. Rev. D 102(4), 046003 (2020). arXiv:2005.14416 [hep-th]

29. N. Evans, M.J. Russell, Low $\mu$ and imaginary $\mu$ signals of a critical point in the phase diagram of an exactly soluble chiral symmetry breaking theory. Phys. Rev. D 102(4), 046018 (2020). arXiv:2006.02117 [hep-th]

30. X. Cao, H. Liu, D. Li, G. Ou, QCD phase diagram at finite isospin chemical potential and temperature in an IR-improved soft-wall AdS/QCD model. Chin. Phys. C 44(8), 083106 (2020). arXiv:2001.02888 [hep-ph]
31. S. He, Y. Yang, P.H. Yuan, Analytic study of magnetic catalysis in holographic QCD. arXiv:2004.01965 [hep-th]

32. X. Cao, H. Liu, D. Li, Pion quasiparticles and QCD phase transitions at finite temperature and isospin density from holography. arXiv:2009.00289 [hep-ph]

33. A. Ballon-Bayona, H. Boschi-Filho, E.F. Capossoli, D.M. Rodrigues, Criticality from Einstein-Maxwell-dilaton holography at finite temperature and density. Phys. Rev. D 102(12), 126003 (2020). arXiv:2006.08810 [hep-th]

34. L.A.H. Mamani, C.V. Flores, V.T. Zanchin, Phase diagram and compact stars in a holographic QCD model. Phys. Rev. D 102(6), 066006 (2020). arXiv:2006.09401 [hep-th]

35. N.R.F. Braga, R. Da Mata, Quasinormal modes for heavy vector mesons in a finite density plasma. Phys. Lett. B 804, 135381 (2020). arXiv:1910.13498 [hep-ph]

36. N.R.F. Braga, R. da Mata, Configuration entropy for quarkonium in a finite density plasma. Phys. Rev. D 101(10), 105016 (2020). arXiv:2002.09413 [hep-th]

37. D.M. Rodrigues, D. Li, E. Folco Capossoli, H. Boschi-Filho, Finite density effects on chiral symmetry breaking in a magnetic field in 2+1 dimensions from holography. arXiv:2010.06762 [hep-th]

38. X. Chen, L. Zhang, D. Li, D. Hou, M. Huang, Gluodynamics and deconfinement phase transition under rotation from holography. arXiv:2010.14478 [hep-ph]

39. A. Ballon-Bayona, J.P. Shock, D. Zoakos, Magnetic catalysis and the chiral condensate in holographic QCD. JHEP 10, 193 (2020). arXiv:2005.00500 [hep-th]

40. J. de Boer, V.E. Hubeny, M. Rangamani, M. Shigemori, Brownian motion in AdS/CFT. JHEP 07, 094 (2009). arXiv:0812.5112 [hepth]

41. A.N. Atmaja, J. de Boer, M. Shigemori, Holographic Brownian motion and time scales in strongly coupled plasmas. Nucl. Phys. B 880, 23-75 (2014). arXiv:1002.2429 [hep-th]

42. S. Chakrabortty, S. Chakraborty, N. Haque, Brownian motion in strongly coupled, anisotropic Yang-Mills plasma: a holographic approach. Phys. Rev. D 89(6), 066013 (2014). arXiv:1311.5023 [hep-th]

43. J. Sadeghi, B. Pourhassan, F. Pourasadollah, Holograghic Brownian motion in $2+1$ dimensional hairy black holes. Eur. Phys. J. C 74(3), 2793 (2014). arXiv:1312.4906 [hep-th]

44. P. Banerjee, B. Sathiapalan, Holographic Brownian motion in 1+1 dimensions. Nucl. Phys. B 884, 74-105 (2014). arXiv:1308.3352 [hep-th]

45. P. Banerjee, Holographic Brownian motion at finite density. Phys. Rev. D 94(12), 126008 (2016). arXiv: 1512.05853 [hep-th]

46. B. Chakrabarty, J. Chakravarty, S. Chaudhuri, C. Jana, R. Loganayagam, A. Sivakumar, Nonlinear Langevin dynamics via holography. JHEP 01, 165 (2020). arXiv:1906.07762 [hep-th]

47. D. Tong, K. Wong, Fluctuation and dissipation at a quantum critical point. Phys. Rev. Lett. 110(6), 061602 (2013). arXiv:1210.1580 [hep-th]

48. M. Edalati, J.F. Pedraza, W. Tangarife Garcia, Quantum fluctuations in holographic theories with hyperscaling violation. Phys. Rev. D 87(4), 046001 (2013). arXiv:1210.6993 [hep-th]

49. E. Kiritsis, Lorentz violation, gravity, dissipation and holography. JHEP 01, 030 (2013). arXiv:1207.2325 [hep-th]

50. W. Fischler, P.H. Nguyen, J.F. Pedraza, W. Tangarife, Fluctuation and dissipation in de Sitter space. JHEP 08, 028 (2014). arXiv: 1404.0347 [hep-th]

51. D. Roychowdhury, Quantum fluctuations and thermal dissipation in higher derivative gravity. Nucl. Phys. B 897, 678-696 (2015). arXiv: 1506.04548 [hep-th]

52. P. Banerjee, B. Sathiapalan, Zero temperature dissipation and holography. JHEP 04, 089 (2016). arXiv:1512.06414 [hep-th] 
53. D. Giataganas, D.S. Lee, C.P. Yeh, Quantum fluctuation and dissipation in holographic theories: a unifying study scheme. JHEP $\mathbf{0 8}$, 110 (2018). arXiv:1802.04983 [hep-th]

54. N.G. Caldeira, E. Folco Capossoli, C.A.D. Zarro, H. Boschi-Filho, Fluctuation and dissipation from a deformed string/gauge duality model. Phys. Rev. D 102(8), 086005 (2020). arXiv:2007.00160 [hep-th]

55. N.G. Caldeira, E. Folco Capossoli, C.A.D. Zarro, H. BoschiFilho, Fluctuation and dissipation within a deformed holographic model with backreaction. Phys. Lett. B 815, 136140 (2021). arXiv:2010.15293 [hep-th]

56. S.S. Gubser, Drag force in AdS/CFT. Phys. Rev. D 74, 126005 (2006). arXiv:hep-th/0605182

57. S.S. Gubser, Comparing the drag force on heavy quarks in $\mathrm{N}=$ 4 super-Yang-Mills theory and QCD. Phys. Rev. D 76, 126003 (2007). arXiv:hep-th/0611272

58. O. Andreev, Drag force on heavy quarks and spatial string tension. Mod. Phys. Lett. A 33(06), 1850041 (2018). arXiv:1707.05045 [hep-ph]

59. O. Andreev, Drag force on heavy diquarks and gauge/string duality. Phys. Rev. D 98(6), 066007 (2018). arXiv:1804.09529 [hep-ph]

60. S. Diles, M.A. Martin Contreras, A. Vega, Drag force in the vacuum of confining gauge theories. arXiv:1912.04948 [hep-th]

61. Y. Kinar, E. Schreiber, J. Sonnenschein, N. Weiss, Quantum fluctuations of Wilson loops from string models. Nucl. Phys. B 583, 76-104 (2000). arXiv:hep-th/9911123

62. U. Gursoy, E. Kiritsis, L. Mazzanti, F. Nitti, Langevin diffusion of heavy quarks in non-conformal holographic backgrounds. JHEP 12, 088 (2010). arXiv:1006.3261 [hep-th]

63. D. Giataganas, H. Soltanpanahi, Heavy quark diffusion in strongly coupled anisotropic plasmas. JHEP 06, 047 (2014). arXiv:1312.7474 [hep-th]

64. D. Giataganas, H. Soltanpanahi, Universal properties of the Langevin diffusion coefficients. Phys. Rev. D 89(2), 026011 (2014). arXiv:1310.6725 [hep-th]

65. D. Dudal, T.G. Mertens, Melting of charmonium in a magnetic field from an effective AdS/QCD model. Phys. Rev. D 91, 086002 (2015). arXiv:1410.3297 [hep-th]

66. D. Dudal, T.G. Mertens, Holographic estimate of heavy quark diffusion in a magnetic field. Phys. Rev. D 97(5), 054035 (2018). arXiv:1802.02805 [hep-th]

67. A. Chamblin, R. Emparan, C.V. Johnson, R.C. Myers, Charged AdS black holes and catastrophic holography. Phys. Rev. D 60, 064018 (1999). arXiv:hep-th/9902170

68. A. Chamblin, R. Emparan, C.V. Johnson, R.C. Myers, Holography, thermodynamics and fluctuations of charged AdS black holes. Phys. Rev. D 60, 104026 (1999). arXiv:hep-th/9904197

69. D. Li, S. He, M. Huang, Q.S. Yan, Thermodynamics of deformed $\mathrm{AdS}_{5}$ model with a positive/negative quadratic correction in graviton-dilaton system. JHEP 09, 041 (2011). arXiv:1103.5389 [hep-th]

70. R.G. Cai, S. He, D. Li, A hQCD model and its phase diagram in Einstein-Maxwell-Dilaton system. JHEP 1203, 033 (2012). arXiv:1201.0820 [hep-th]

71. S. He, S.Y. Wu, Y. Yang, P.H. Yuan, Phase structure in a dynamical soft-wall holographic QCD model. JHEP 04, 093 (2013). arXiv:1301.0385 [hep-th]

72. D. Li, J. Liao, M. Huang, Enhancement of jet quenching around phase transition: result from the dynamical holographic model. Phys. Rev. D 89(12), 126006 (2014). arXiv:1401.2035 [hep-ph]

73. Y. Yang, P.H. Yuan, A refined holographic QCD model and QCD phase structure. JHEP 11, 149 (2014). https://doi.org/10.1007/ JHEP11(2014)149. arXiv:1406.1865 [hep-th]

74. Z. Li, Y. Chen, D. Li, M. Huang, Locating the QCD critical end point through the peaked baryon number susceptibilities along the freeze-out line. Chin. Phys. C 42(1), 013103 (2018). arXiv:1706.02238 [hep-ph]

75. X. Chen, D. Li, M. Huang, Criticality of QCD in a holographic QCD model with critical end point. Chin. Phys. C 43(2), 023105 (2019). arXiv:1810.02136 [hep-ph]

76. I. Aref'eva, K. Rannu, Holographic anisotropic background with confinement-deconfinement phase transition. JHEP 05, 206 (2018). https://doi.org/10.1007/JHEP05(2018)206. arXiv:1802.05652 [hep-th]

77. X. Chen, D. Li, D. Hou, M. Huang, Quarkyonic phase from quenched dynamical holographic QCD model. JHEP 03, 073 (2020). arXiv:1908.02000 [hep-ph]

78. I. Aref'eva, K. Rannu, P. Slepov, Orientation dependence of confinement-deconfinement phase transition in anisotropic media. Phys. Lett. B 792, 470-475 (2019). arXiv:1808.05596 [hep-th]

79. P. Colangelo, F. De Fazio, N. Losacco, Chaos in a $Q \bar{Q}$ system at finite temperature and baryon density. Phys. Rev. D 102(7), 074016 (2020). arXiv:2007.06980 [hep-ph]

80. H. Bohra, D. Dudal, A. Hajilou, S. Mahapatra, Chiral transition in the probe approximation from an Einstein-Maxwell-dilaton gravity model. arXiv:2010.04578 [hep-th]

81. A. Ballon-Bayona, H. Boschi-Filho, L.A.H. Mamani, A.S. Miranda, V.T. Zanchin, Effective holographic models for QCD: glueball spectrum and trace anomaly. Phys. Rev. D 97(4), 046001 (2018). arXiv:1708.08968 [hep-th]

82. O. Andreev, $1 / \mathrm{q}^{* *} 2$ corrections and gauge/string duality. Phys. Rev. D 73, 107901 (2006). arXiv:hep-th/0603170

83. O. Andreev, V.I. Zakharov, Heavy-quark potentials and AdS/QCD. Phys. Rev. D 74, 025023 (2006). arXiv:hep-ph/0604204

84. A. Karch, E. Katz, D.T. Son, M.A. Stephanov, Linear confinement and AdS/QCD. Phys. Rev. D 74, 015005 (2006). [arXiv:hep-ph/0602229 [hep-ph]]

85. S.S. Afonin, Generalized soft wall model. Phys. Lett. B 719, 399403 (2013). arXiv:1210.5210 [hep-ph]

86. M. Rinaldi, V. Vento, Scalar and tensor glueballs as gravitons. Eur. Phys. J. A 54, 151 (2018). arXiv:1710.09225 [hep-ph]

87. S. Diles, Probing AdS/QCD backgrounds with semi-classical strings. EPL 130(5), 51001 (2020). arXiv:1811.03141 [hep-th]

88. S. Afonin, A. Katanaeva, Glueballs and deconfinement temperature in AdS/QCD. Phys. Rev. D 98(11), 114027 (2018). arXiv:1809.07730 [hep-ph]

89. R.C.L. Bruni, E. Folco Capossoli, H. Boschi-Filho, Quarkantiquark potential from a deformed AdS/QCD. Adv. High Energy Phys. 2019, 1901659 (2019). arXiv: 1806.05720 [hep-th]

90. E. FolcoCapossoli, M.A. Martín Contreras, D. Li, A. Vega, H. Boschi-Filho, Hadronic spectra from deformed AdS backgrounds. Chin. Phys. C 44(6), 064104 (2020). arXiv:1903.06269 [hep-ph]

91. M. Rinaldi, V. Vento, Scalar spectrum in a graviton soft wall model. J. Phys. G 47(12), 125003 (2020). arXiv:2002.11720 [hep-ph]

92. E. Folco Capossoli, M.A. Martín Contreras, D. Li, A. Vega, H. Boschi-Filho, Proton structure functions from an AdS/QCD model with a deformed background. Phys. Rev. D 102(8), 086004 (2020). arXiv:2007.09283 [hep-ph]

93. M.A.M. Contreras, E.F. Capossoli, D. Li, A. Vega, H. BoschiFilho, Pion form factor from an AdS deformed background. arXiv:2104.04640 [hep-ph]

94. M.A.M. Contreras, E.F. Capossoli, D. Li, A. Vega, H. Boschi-Filho, Proton and neutron form factors from deformed gravity/gauge duality. Phys. Lett. B 822, 136638 (2021). arXiv:2108.05427 [hep-ph]

95. D.N. Zubarev, Nonequilibrium statistical thermodynamics (Plenum Press, New York, 1974)

96. Y.A. Markov, M.A. Markova, On the fluctuation-dissipation theorem for soft fermionic excitations in a hot QCD plasma. Nucl. Phys. A 840, 76-96 (2010). arXiv:0909.0377 [hep-ph] 
97. S.A. Hartnoll, Lectures on holographic methods for condensed matter physics. Class. Quantum Gravity 26, 224002 (2009). arXiv:0903.3246 [hep-th]

98. R. Wald, Quantum field theory in curved spacetime and black hole thermodynamics (The University of Chicago Press, Chicago, 1994)
99. A. Bodrova, A. Chechkin, A. Cherstvy et al., Underdamped scaled Brownian motion: (non-) existence of the overdamped limit in anomalous diffusion. Sci. Rep. 6, 30520 (2016) 